\title{
The ATF/CREB Transcription Factor Atf1 Is Essential for Full Virulence, Deoxynivalenol Production, and Stress Tolerance in the Cereal Pathogen Fusarium graminearum
}

\author{
Thuat Van Nguyen, Cathrin Kröger, Jakob Bönnighausen, Wilhelm Schäfer, and Jörg Bormann \\ University of Hamburg, Biocenter Klein Flottbek, Department of Molecular Phytopathology and Genetics, Ohnhorststr. 18, \\ D-22609 Hamburg, Germany
}

Submitted 30 April 2013. Accepted 23 July 2013.

Fusarium graminearum is a necrotrophic plant pathogen of cereals that produces mycotoxins such as deoxynivalenol (DON) and zearalenone (ZEA) in grains. The stress-activated mitogen-activated protein kinase FgOS-2 is a central regulator in $F$. graminearum and controls, among others, virulence and DON and ZEA production. Here, we characterized the ATF/CREB-activating transcription factor FgAtf1, a regulator that functions downstream of FgOS-2. We created deletion and overexpression mutants of Fgatf1, the latter being also in an $\mathrm{FgOS}$-2 deletion mutant. FgAtf1 localizes to the nucleus and appears to interact with FgOS2 under osmotic stress conditions. Deletion mutants in Fgatf1 ( $\Delta F$ Fatf1) are more sensitive to osmotic stress and less sensitive to oxidative stress compared with the wild type. Furthermore, sexual reproduction is delayed. $\Delta$ Fgatf1 strains produced higher amounts of DON under in vitro induction conditions than that of the wild type. However, during wheat infection, DON production by $\Delta F$ gatf1 is strongly reduced. The $\Delta$ Fgatf1 strains displayed strongly reduced virulence to wheat and maize. Interestingly, constitutive expression of Fgatf1 in the wild type led to hypervirulence on wheat, maize, and Brachypodium distachyon. Moreover, constitutive expression of $\mathrm{Fgatf1}$ in the $\triangle \mathrm{FgOS}-2$ mutant background almost complements $\triangle F g O S$-2-phenotypes. These data suggest that FgAtf1 may be the most important transcription factor regulated by FgOS-2.

Viability of every living entity depends on rapid adaptation to environmental changes. Sensing of external stimuli and subsequent cellular responses mainly rely on mitogen-activated protein (MAP) kinase phosphorelay systems such as the conserved stress-activated protein kinase (SAPK) pathway. In previous studies, we and others demonstrated the preeminent importance of SAPK signaling for stress adaptation, sexual reproduction, and virulence (Heller et al. 2012; Kawasaki et al. 2002; Nguyen et al. 2012; Segmüller et al. 2007). Disruption of FgOS-2, the Fusarium graminearum homolog of the Saccharomyces cerevisiae high osmolarity glycerol (HOG) kinase, impaired vegetative growth, sexual reproduction, osmotic stress adaptation, sec-

Corresponding author: J. Bormann; Telephone: +49 4042816 309, E-mail: bormannj@botanik.uni-hamburg.de

* The $\boldsymbol{e}$-Xtra logo stands for "electronic extra" and indicates that 12 supplementary figures and five supplementary tables are published online.

(C) 2013 The American Phytopathological Society ondary metabolism, and, most strikingly, pathogenicity toward wheat and maize. We showed that most of these phenotypes could be connected to a disturbed reactive oxygen species (ROS) metabolism (Nguyen et al. 2012). In general, ROS are regarded as harmful by-products of numerous physiological processes (Apel and Hirt 2004) but they also facilitate an immediate response to pathogen attack. At the site of infection, localized ROS production by plant peroxidases leads to cell wall enforcement (Passardi et al. 2004). Furthermore, ROS exhibit antimicrobial activity toward the invading pathogen. In addition, ROS may act as a second messenger. It has been reported that ROS production regulates the production of numerous proteins involved in host defense reactions or apoptosis (Gadjev et al. 2008; Gechev et al. 2006; Orozco-Cárdenas et al. 2001). However, for successful penetration and host colonization, fungal pathogens evolved strategies to deal with oxidative stress (Heller and Tudzynski 2011). Biotrophic pathogens manage to detoxify plant-derived ROS by inducible catabolic enzymes (e.g., catalases and superoxide dismutases) (Molina and Kahmann 2007) or by secretion of ROS quencher (Voegele et al. 2005). Pathogenic development of necrotrophic fungi may depend on the hypersensitive response of the plant. It is feasible that some necrotrophic fungi (i.e., Botrytis cinerea) (Govrin and Levine 2000) even actively contribute to plant-derived ROS by secretion of effectors (El Oirdi et al. 2011). Data obtained from studies in Schizosaccharomyces pombe involve SAPK-signaling cascades in the perception of oxidative stress and the regulation of ROSresponsive genes (Buck et al. 2001; Nguyen et al. 2000). These cascades consist of one or more sensor kinases, one or two response regulators, and the MAP kinase relay (three histidine kinases: MAP kinase-kinase-kinase, MAP kinase-kinase, and MAP kinase), which is conserved throughout all kingdoms. A putative downstream target of the SAPK-cascade is supposed to be the ATF/CREB transcription factor Atf1. In S. pombe, Atf1 is activated by $\mathrm{Spc} 1 / \mathrm{Sty} 1$, the FgOS-2 homolog. After activation, it regulates the expression of a set of $\mathrm{H}_{2} \mathrm{O}_{2}$-responsive genes (Chen et al. 2003). Interestingly, the function of Atf1 homologs in phytopathogenic fungi is not conserved. Thus, their importance for disease development varies. Knock-out mutants of the $\mathrm{B}$. $\mathrm{ci}$ nerea Atf1 are hypervirulent toward different host plants. A comparative microarray approach in $B$. cinerea revealed a high number of genes regulated by Atf1. Among them are numerous genes involved in secondary metabolism (Temme et al. 2012). In the phytopathogenic fungus Magnaporthe oryzae, Atf1 mutants show a reduced virulence toward rice leaves, mainly because the plant defense was elevated compared with a wild-type infection (Guo et al. 2010). Furthermore, MoAtf1 plays a major role in the response to oxidative stress mediated by $\mathrm{H}_{2} \mathrm{O}_{2}$. Obviously, in 
the mutant strains, the capabilities for ROS degradation are compromised. In the biotrophic grass pathogen Claviceps purpurea, the Atf1 homolog TF1 is believed to act as a positive regulator of catalase genes and a repressor of nox genes (Nathues et al. 2004). In TF1 deletion mutants, virulence against rye is attenuated. In view of this diversity in function among phytopathogenic fungi, it is of special interest to investigate the Atf1 homolog in $F$. graminearum. The filamentous ascomycete $F$. graminearum is a highly organ-specific pathogen that resides on small grain cereals such as rice, wheat, and barley, entering its host through the flower at anthesis. Maize is infected through the silk channel or through wounds of the ears. Once the host plants are colonized, crops become unsuitable for food and feed production. This is mainly due to massive mycotoxin accumulation but also to reductions in grain development. The most devastating mycotoxins produced by $F$. graminearum are deoxynivalenol (DON) and zearalenone (ZEA). The trichothecene DON acts as a potent inhibitor of the eukaryotic translational machinery (Rocha et al. 2005). ZEA, a polyketide, shares some structural similarities to estrogen and thereby compromises the health of women and their unborn children (Kuiper-Goodman et al. 1987). The regulation of mycotoxin production is not yet fully understood. Several in vitro studies describe numerous factors that influence the regulation of mycotoxin biosynthesis. The production of DON was shown to be repressed under salt stress (Ochiai et al. 2007) and induced under oxidative stress conditions. Furthermore, the $\mathrm{pH}$ value (Gardiner et al. 2009c), nitrogen (Gardiner et al. 2009a), carbon source (Jiao et al. 2008), and even cobalt chloride (Tsuyuki et al. 2011) were found to influence DON production in vitro. However, there seem to be at least two distinct signaling cascades that regulate the production of DON. We could clearly demonstrate that $\mathrm{FgOS}-2$ deletion leads to an increased DON production under in vitro conditions and, on the other hand, to a strong decrease in DON production during wheat infection compared with the wild type. Fgatfl transcript levels are subject to the upstream kinase FgOS-2 (Nguyen et al. 2012). In the $\Delta F g O S$-2 deletion strains, Fgatfl is strongly downregulated during wheat infection and upregulated under oxidative stress conditions. In this study, we functionally characterize the $F$. graminearum Atf1 homolog in regard to its pivotal role in stress adaptation, secondary metabolism (including mycotoxin production), sexual reproduction, and virulence toward different hosts. Under stress conditions, FgAtf1 and FgOS-2 physically interact in the nucleus. Furthermore, results obtained from overexpression experiments indicate that FgAtf1 is the main downstream target of FgOS-2.

\section{RESULTS}

\section{Generation of mutants.}

In a genome-wide survey for regulatory motifs in the genome of $F$. graminearum, Kumar and co-workers describe an Atf1 ortholog (accession number FGSG_10142) according to its target regulatory motif ACGTCA. According to the Munich Information Center for Protein Sequences Fusarium graminearum genome database (Wong et al. 2010), the protein consists of 526 amino acids. Prosite database (Sigrist et al. 2010) analysis revealed that the putative FgAtfl ortholog contains a basic-leucine zipper domain for DNA binding ranging from amino acid K407 to T489 (Supplementary Fig. S1).

In order to study the function of this protein in the life cycle of $F$. graminearum, the gene was deleted using a gene-replacement approach (Supplementary Fig. S2A). Fifty primary transformants were screened in a diagnostic polymerase chain reaction (PCR) (data not shown). Five independent transformants showed homologous integration of the replacement fragment (Supplementary Table S1). Single conidia of those mutants were used to obtain homokaryotic deletion mutants. One additional mutant was chosen which showed amplification of the wildtype gene even after repetitive single-spore isolations, indicating an ectopic integration of the vector. All of the selected mutants and the wild type were analyzed in a Southern blot experiment. When probed for the integration of the hygromycin gene, all mutants but not the wild type showed a single integration of the fragment. When hybridized with a probe amplified from the Fgatfl gene, all mutants, except the one expected to have an ectopic integration of the replacement construct, showed no signal. In the wild type, a signal was visible. One $\Delta F$ gatfl strain (number 13.2.) was subsequently selected for fluorescent labeling using the red fluorescent protein dsRed (discussed below). For localization studies, mutants carrying a C-terminal fusion of the open reading frame (ORF) of Fgatfl with the red fluorescent protein mCherry were generated. Additionally, mutants were generated that constitutively express Fgatfl under the control of the glyceraldehyde 3-phosphate dehydrogenase (gpdA) promoter from Cochliobolus heterostrophus (Salomon et al. 2012) (Supplementary Fig. S3A). Two independent transformants were obtained from this transformation (from now on referred to as Fgatfl $^{\text {oe }}$ mutant strains). One of those mutants showed a 16-fold upregulation of Fgatfl transcript level compared with the wild type when raised for 4 days in liquid complete medium (CM) and a 15-fold upregulation during wheat head infection (10 days postinoculation [dpi]). In this particular Fgatfl overexpression mutant, $F g O S$-2 was deleted using the methods described previously (Nguyen et al. 2012) (Supplementary Fig. S4A). Seven mutants with a disrupted FgOS-2

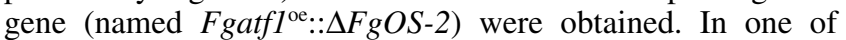
these mutants (1346.1.4), Fgatfl transcript level was determined to verify whether the Fgatfl transcript level is influenced by the deletion of FgOS-2. During growth in CM and during wheat infection, Fgatfl transcript level was elevated 12 and 14 times, respectively, compared with the wild type.

\section{Functional FgAtf1 is necessary for proper vegetative growth and interacts with FgOS-2 under osmotic stress conditions.}

To test the influence of FgAtf1 on vegetative growth, plate assays on different media were conducted using three independent $\Delta$ Fgatfl mutants, an ectopic mutant, and the wild type. When grown on CM agar plates, Fgatfl deletion mutants were slightly reduced in growth (Fig. 1A) compared with the wild type. On agar plates supplemented with osmotic agents $(1.2 \mathrm{M}$ sorbitol, $0.8 \mathrm{M} \mathrm{KCl}$, and $0.8 \mathrm{M} \mathrm{NaCl}$ ), the growth reduction was more pronounced. However, in contrast to $\mathrm{FgOS}-2$ deletion strains, growth was still possible on plates containing 0.8 $\mathrm{M} \mathrm{NaCl}$ (Fig. 1A and B) (Nguyen et al. 2012). Also, other concentrations of $\mathrm{NaCl}$ and sorbitol $(0.3$ and $0.2 \mathrm{M})$ and other salts (such as $\mathrm{MgCl}_{2}, \mathrm{CaCl}_{2}, \mathrm{LiCl}, \mathrm{ZnCl}_{2}$, and $\mathrm{FeCl}_{3}$ ) were tested in a plate assay. On the concentrations tested, the $\triangle F g O S-2$ showed a growth reduction on all media tested, whereas the Fgatfl deletion strain was most sensitive to sorbitol, $\mathrm{LiCl}$, and $\mathrm{ZnCl}_{3}$ (Supplementary Fig. S5).

Conidial germination of $\Delta F$ gatfl mutants also differed from the wild type under salt stress conditions. In the presence of $0.8 \mathrm{M} \mathrm{NaCl}$, germ tubes usually emerged at one apical compartment of $\Delta F$ gatfl conidia. The wild type, in contrast, usually germinated out of two compartments. Under nonstress conditions, conidial germination was similar between the strains (Supplementary Fig. S6). Compared with the wild type, detection of ROS using nitro blue tetrazolium chloride (NBT) revealed no remarkable coloration of the colonies of all Fgatfl mutants on $\mathrm{CM}$ containing 0.2 and $0.8 \mathrm{M} \mathrm{NaCl}$. In contrast, the $\mathrm{FgOS}$-2 deletion strain showed a strong coloration when grown on $0.2 \mathrm{M} \mathrm{NaCl}$ (Nguyen et al. 2012) (Supplementary 
Fig. S7). Hence, there is no accumulation of ROS under osmotic stress conditions in the $\Delta$ Fgatfl strains. The Fgatfl $^{\text {oe }}$ mutant strains (two independent mutants were tested) also showed a slightly reduced growth on the osmotic stress medium $(0.8 \mathrm{M}$ $\mathrm{NaCl}$ ) but grew better than the Fgatfl deletion strains. Interestingly, the $F g a t f 1^{\text {oe }:: \Delta F g O S-2}$ mutants (three independent mutants were tested) grew much better than the $\Delta F g O S-2 \mathrm{mu}$ tant strains on medium conferring osmotic stress (Fig. 1B). On medium containing $0.3 \mathrm{M} \mathrm{NaCl}$, overexpression of Fgatfl led to a nearly full restoration of growth in the $\Delta F g O S-2$ mutant background (Fig. 1B). The same applies for the growth on other metals. Accordingly, these mutants also accumulated less ROS compared with the $\triangle F g O S$ - 2 mutants: staining with NBT revealed wild-type like coloration at the growing edge of the Fgatfl $^{\mathrm{oe}}:: \Delta F g O S-2$ colonies on $\mathrm{CM}$ containing $0.2 \mathrm{M} \mathrm{NaCl}$.
Unlike the $F g O S$-2 deletion strains, the $\Delta F g$ atfl mutants exhibited no resistance to the phenylpyrrolic fungicide fludioxonil (Supplementary Fig. S8). The Fgatfl ${ }^{\mathrm{oe}}: \Delta F g O S$-2 mutant exhibited the highest resistance to fludioxonil. However, also on CM, the Fgatf $1^{\text {oe }}:: \Delta F g O S-2$ mutants grew faster than the $\triangle F g O S$-2 mutants (Fig. 1B). These results indicate that FgAtf1 is a downstream target of FgOS-2 under osmotic stress and that activation of FgAtf1 is necessary for proper vegetative growth. Furthermore, fludioxonil seems not to act on FgAtf1.

The Fgatfl deletion strains, not the overexpression mutants, grew much faster on medium supplemented with $\mathrm{H}_{2} \mathrm{O}_{2}$ compared with the wild type (Fig. 2). $\mathrm{FgOS}$-2 deletion also leads to an increased resistance to oxidative stress (Nguyen et al. 2012). This phenotype is connected to a regulation of catalase gene expression and activity (discussed below). The increased resis-
A

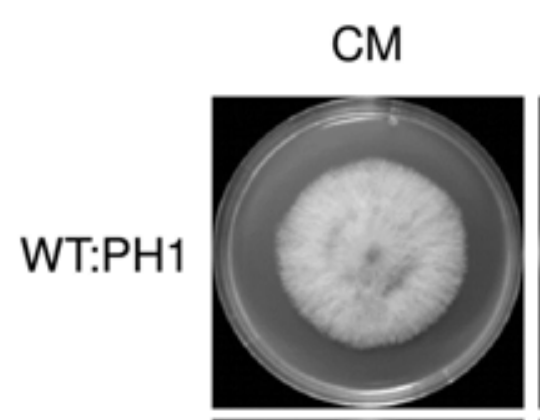

$\Delta$ Fgatf1

Fgatf10e
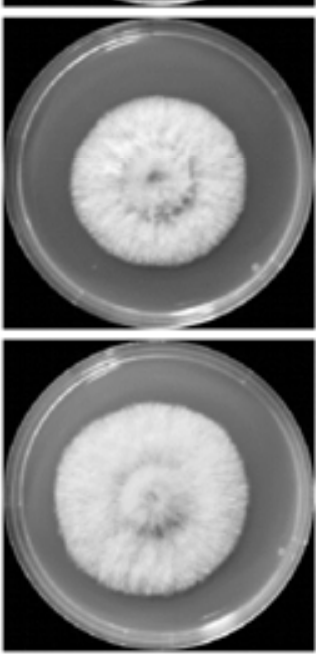

B

\section{Fgatf10e:: $\triangle F g O S-2$} $\mathrm{CM}$

$\triangle F g O S-2$

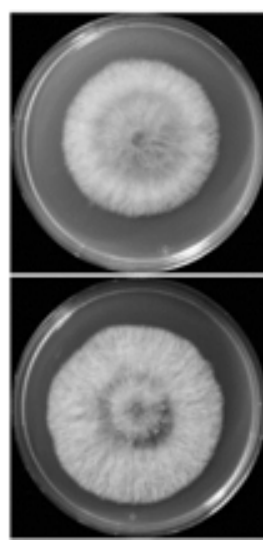

$\mathrm{CM}$ 1.2 M sorbitol
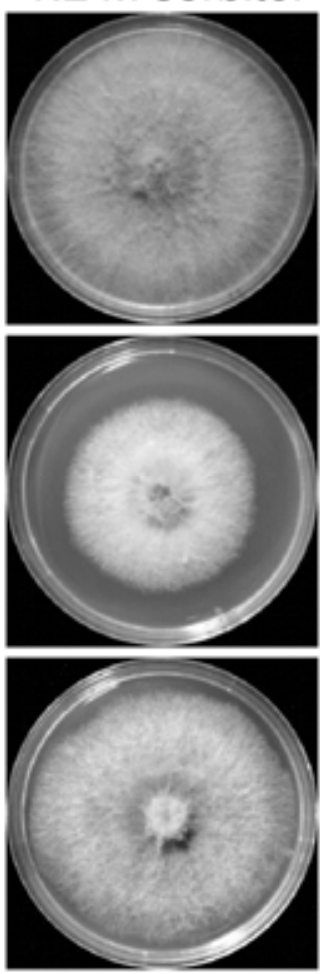

$\mathrm{CM}$
$\mathrm{CM}$ $0.8 \mathrm{M} \mathrm{KCl}$
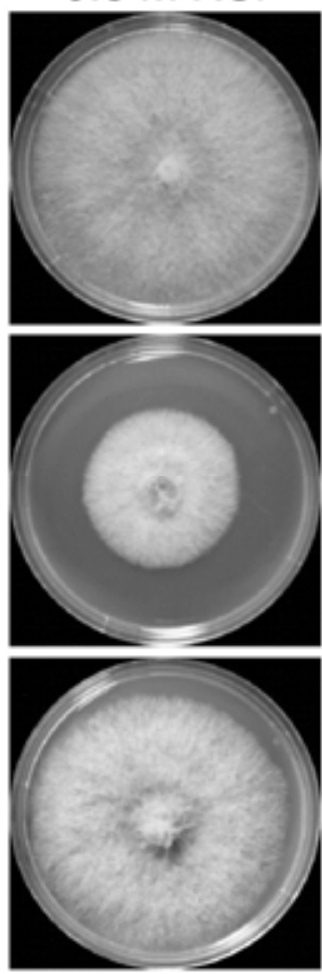

$\mathrm{CM}$
$\mathrm{CM}$
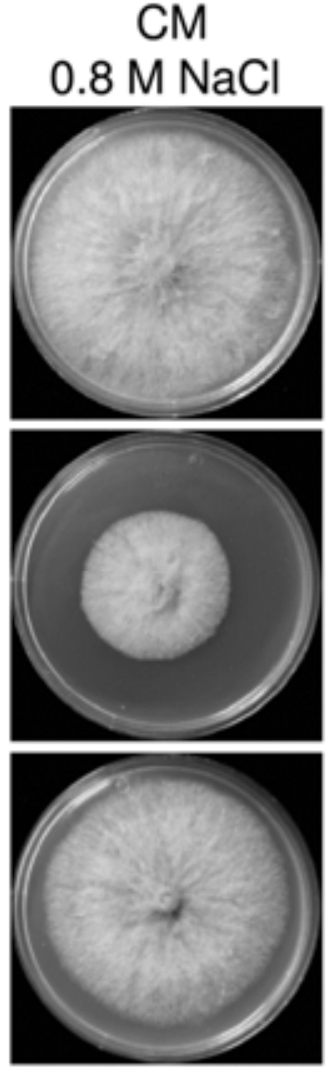

$\mathrm{CM}$ $1.2 \mathrm{M}$ sorbitol $0.8 \mathrm{M} \mathrm{KCl}$ $0.8 \mathrm{M} \mathrm{NaCl}$ $0.3 \mathrm{M} \mathrm{NaCl}$
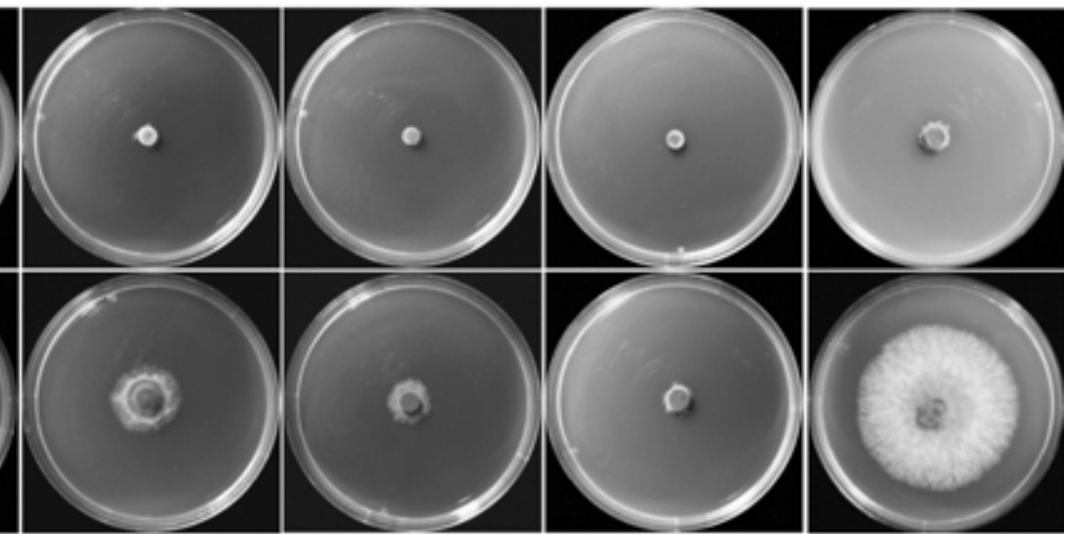

Fig. 1. Colony morphology of $\mathbf{A}$, the wild type (WT:PH1) and $\triangle F g a t f 1$ and $F$ gatf ${ }^{\mathrm{oe}}$ mutants and $\mathbf{B}, \Delta F g O S-2$ and $F g a t f 1^{\text {oe }}: \Delta F g O S-2$ mutants after 3 days of growth on complete medium (CM) and $\mathrm{CM}$ supplemented with $1.2 \mathrm{M}$ sorbitol, $0.8 \mathrm{M} \mathrm{KCl}, 0.8 \mathrm{M} \mathrm{NaCl}$, and $0.3 \mathrm{M} \mathrm{NaCl}$. Agar plates were inoculated with mycelial plugs from 3-day-old cultures. 
tance to $\mathrm{H}_{2} \mathrm{O}_{2}$ substantiates our assumption that the FgOS-2/ FgAtf1-cascade is the central trigger of ROS metabolism in F. graminearum.

To further prove the interaction of FgOS-2 and FgAtf1 under stress conditions, we performed bimolecular fluorescence complementation (BiFC) (Hoff and Kück 2005). For this purpose, the ORF of $F g O S$-2 was fused to the $\mathrm{N}$ terminus of the yellow fluorescent protein (YFP) and the ORF of Fgatfl was fused to the $\mathrm{C}$ terminus of YFP. A strain expressing a histone H1-mCherry fusion which facilitates detection of nuclei (A. L.
Martínez-Rocha and W. Schäfer, unpublished results) was used as recipient for both plasmids. Strains obtained from co-transformation of both plasmids were initially analyzed by PCR (data not shown). Mutants positive for both plasmids were subsequently analyzed by confocal laser-scanning microscopy (discussed below). Unstressed mutant mycelium as well as the wild type did not emit YFP-fluorescence when excited with a 514-nm laser line. Application of $1.2 \mathrm{M} \mathrm{NaCl}$ evoked fluorescence inside the nucleus of fungal hyphae of the mutants within 15 min after application (Fig. 3).

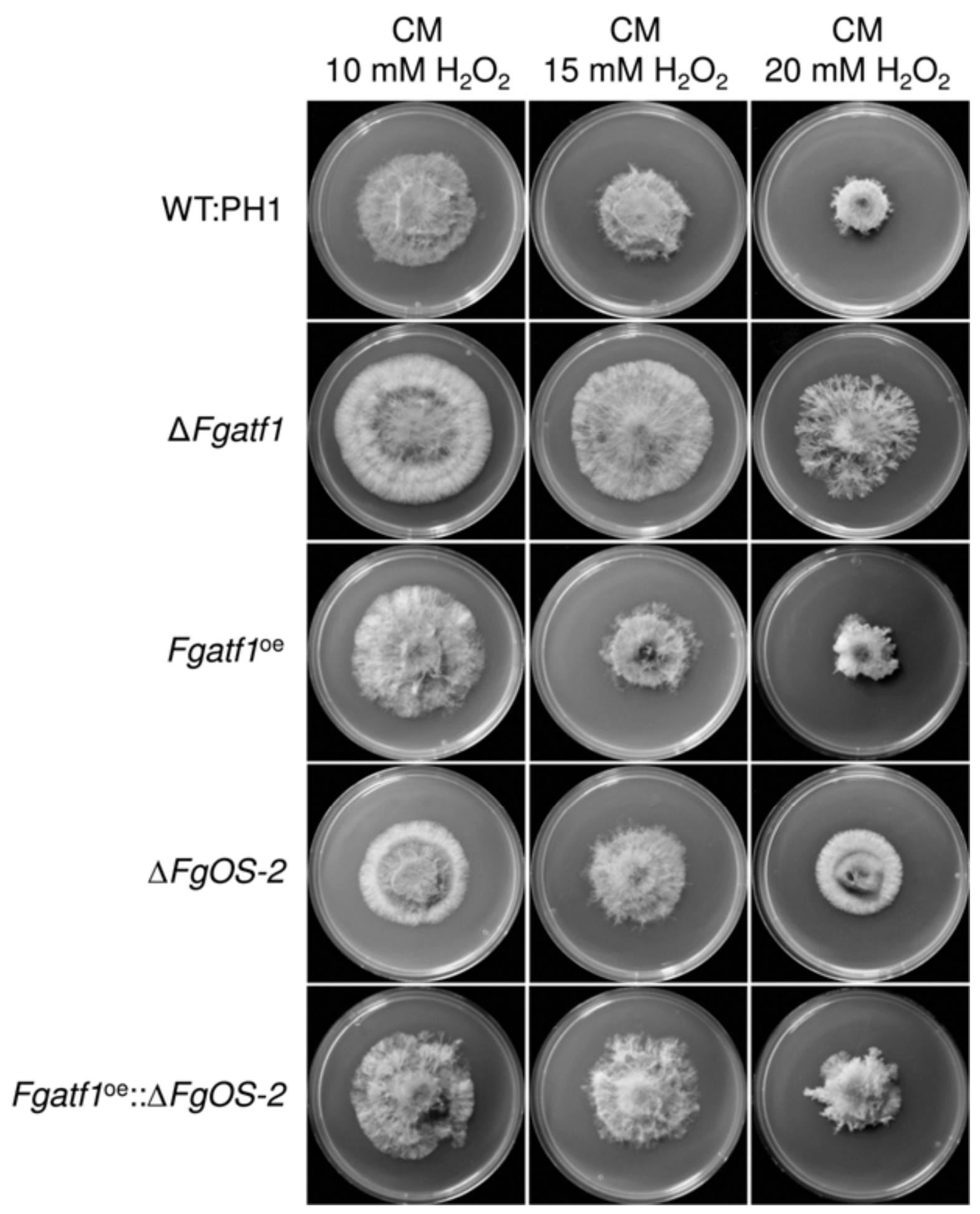

Fig. 2. Colony morphology of the wild type (WT:PH1) and the $\Delta F g a t f 1, F g a t f 1^{\text {oe }}, \Delta F g O S-2$, and $F g a t f 1^{\text {oe }}:: \Delta F g O S$ - 2 mutants after 4 days of growth on complete medium (CM) and CM supplemented with 10, 15, and $20 \mathrm{mM} \mathrm{H}_{2} \mathrm{O}_{2}$. Agar plates were inoculated with mycelial plugs from 3-day-old cultures. 
This result provides further evidence for an interaction of FgAtf1 and FgOS-2 under osmotic stress conditions. Zheng and coworkers (2012) recently reported that FgOS-2 translocates from the cytosol to the nucleus after treatment with $\mathrm{NaCl}$. To elucidate whether FgAtf1 also translocates to the nucleus, we performed live-cell imaging using strains that expressed an FgAtf1-mCherry fusion protein. FgAtf 1 constitutively resides in the nucleus. This localization pattern seems to be independent of FgOS-2, because it was also observed in $\triangle F g O S-2 \mathrm{mu}$ tants. Furthermore, it is independent of osmotic and oxidative stress in both strains (Supplementary Fig. S9).

\section{Constitutive expression of $\mathrm{Fgatf1}$ restores}

sexual reproduction in $\mathrm{FgOS}-2$ deletion strains.

Functional FgOS-2 is necessary for sexual reproduction in $F$. graminearum (Nguyen et al. 2012). To test the importance of FgAtf1 in this process, we analyzed the Fgatfl mutant strains and the $\triangle F g O S$-2 for perithecia formation on both carrot agar and detached wheat nodes (Fig. 4). On both substrates, perithecia formation was delayed in the $\Delta F$ gatfl-strains (three independent mutants were tested). Even after prolonged incubation, approximately $50 \%$ of all perithecia remained immature (Fig. $4 \mathrm{~B}$ and $\mathrm{G})$. Although the wild type usually produces mature perithecia containing numerous asci and ascospores after 3 weeks (Fig. 4A and F), it took 7 and 8 weeks (wheat nodes and carrot agar, respectively) for the $\Delta F g a t f l$-strains. However, those perithecia that finally matured also contained asci and ascospores (Fig. 4B). Ascospores discharged from the perithecia on wheat nodes were viable and gave rise to new colonies (Supplementary Fig. S10). Interestingly, overexpression of Fgatfl restored the ability of an $\mathrm{FgOS}$-2 deletion strain to produce perithecia. Although the $\triangle F g O S-2$ strains completely failed to reproduce sexually (Fig. 4D and I), three independent Fgatf ${ }^{\text {oe.: }}$ $\Delta F g O S$ - 2 mutants produced mature asci and ascospores after 7 and 8 weeks (wheat nodes and carrot agar, respectively) (Fig. 4E and J). However, the clusters of perithecia appeared much smaller and not much aggregated to clusters compared with the wild type. Over expression of Fgatfl in the wild type did not affect perithecia formation (Fig. 4C and $\mathrm{H}$ ).

\section{Deletion of Fgatf1 attenuates virulence toward wheat and maize.}

Along with others, we implicated the FgOS-2 signaling cascade in virulence toward two important hosts of $F$. graminearum, wheat and maize (Jiang et al. 2011; Nguyen et al. 2012). Surprisingly, deletion of Fgatfl resembles neither the apatho-

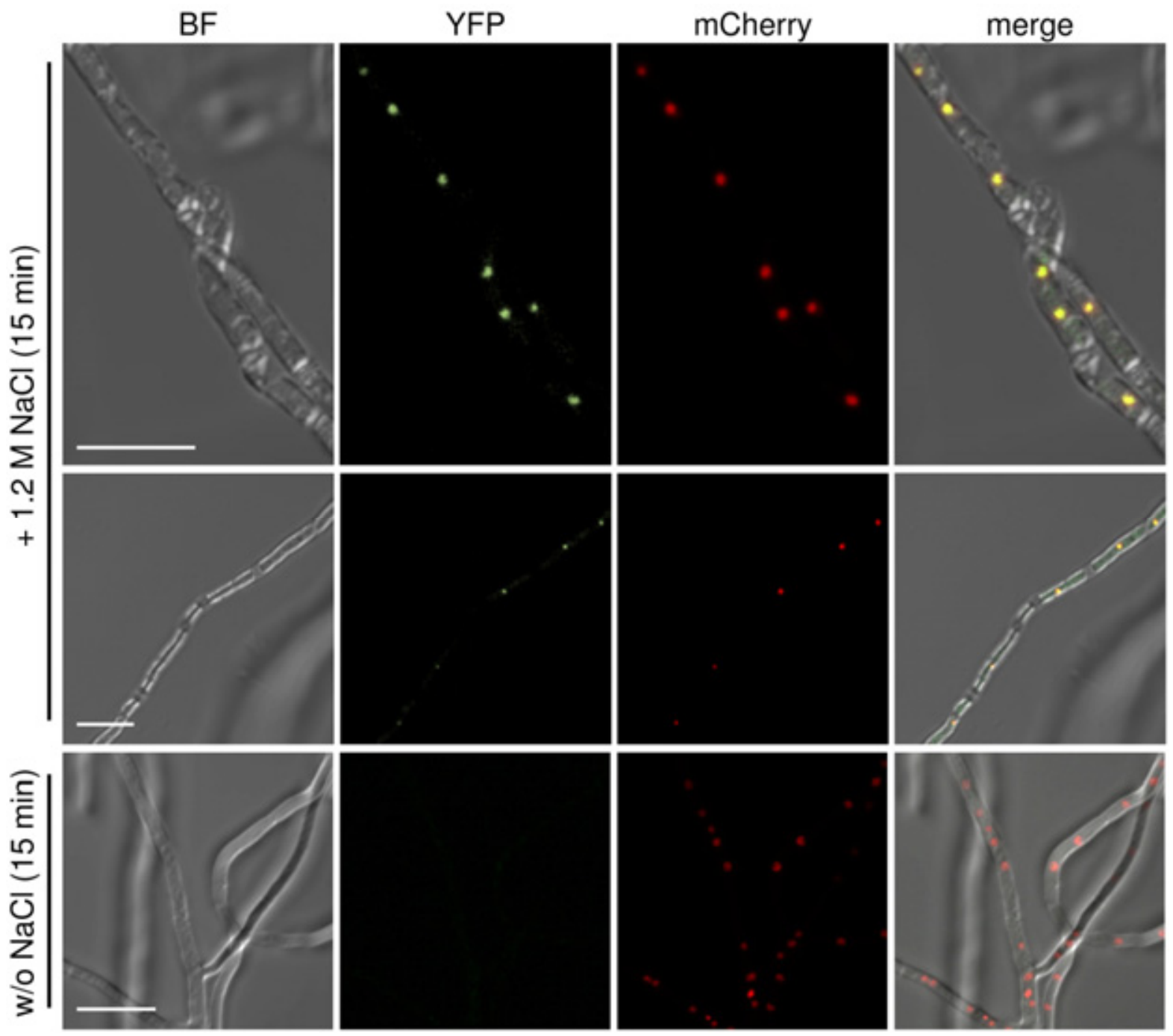

Fig. 3. Bimolecular fluorescence (BF) complementation assay. FgOS-2 was fused to the $\mathrm{N}$ terminus and FgAtf1 to the $\mathrm{C}$ terminus of yellow fluorescent protein (YFP). Both constructs were co-transformed into a Fusarium graminearum strain expressing a histone-1-mCherry fusion protein facilitating visualization of nuclei. At $24 \mathrm{~h}$ after germination of conidia, osmotic stress mediated by $1.2 \mathrm{M} \mathrm{NaCl}$ was applied and hyphae were subsequently imaged using confocal laser-scanning microscopy. Scale bar: $10 \mu \mathrm{m}$. 
genic phenotype of the $F g O S$-2 deletion strains on maize nor the reduced virulence on wheat. The pathogenic potential of $\Delta$ Fgatfl strains (tested with three independent mutants) was reduced compared with the wild type but clearly increased compared with the $\triangle F g O S$-2 strains (Fig. 5). On wheat ('Nandu'), generally, only the point-inoculated spikelet got colonized by the $\Delta$ Fgatf1 strains within $21 \mathrm{dpi}$. However, the colonization occurred faster compared with the $\Delta F g O S-2$ mutant. The

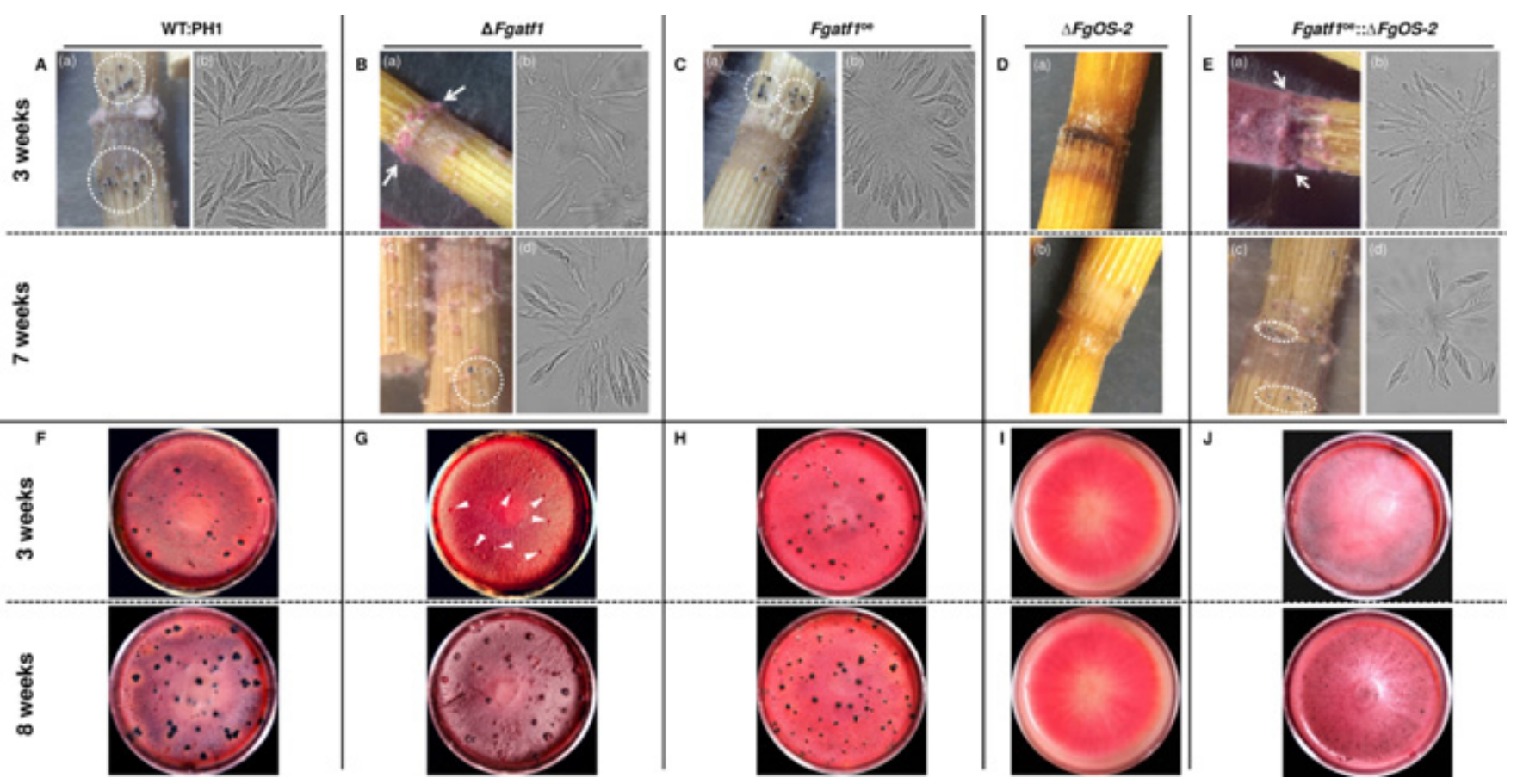

Fig. 4. Perithecia and ascospore development on $\mathbf{A}$ to $\mathbf{E}$, detached wheat nodes and $\mathbf{F}$ to $\mathbf{J}$, carrot agar. Mycelia of $\mathbf{A}$ and $\mathbf{F}$, the wild type (WT:PH1) and the mutants $\mathbf{B}$ and $\mathbf{G}, \Delta F$ gatf $1 ; \mathbf{C}$ and $\mathbf{H}, F$ gatf $1^{\circ} ; \mathbf{D}$ and $\mathbf{I}, \Delta F g O S$ - 2 ; and $\mathbf{E}$ and $\mathbf{J}, F g a t f l^{\text {oe }}: \Delta F g O S$ - 2 were assayed. $\mathbf{A}$ to $\mathbf{E}$, Clusters of perithecia are indicated by the dotted circles in A (a), B (d), C (a), and E (c). Squeezing these clusters released the asci in A (b), B (b and d), C (b), and E (b and d). The $\Delta F g a t f 1$ (arrows in B [a]) and the $F g a t f 1^{\circ}:: \Delta F g O S-2$ (arrows in E [a]) mutants produced immature perithecia clusters, which mature after 56 days of incubation (B [c] and $\mathrm{E}[\mathrm{c}]$ ). F, The wild type and $\mathbf{H}$, the $F g a t f f^{\mathrm{oe}}$ mutants started to produce perithecia after 21 days postinoculation (dpi) on carrot agar. $\mathbf{G}$, After 56 dpi,

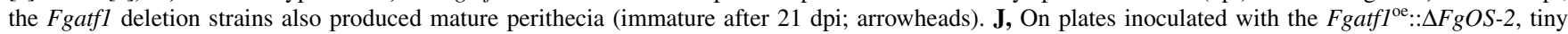
clusters of perithecia became visible after 56 dpi. Deletion of $\mathrm{FgOS}$-2 in the wild type led to a complete loss of perithecia formation.
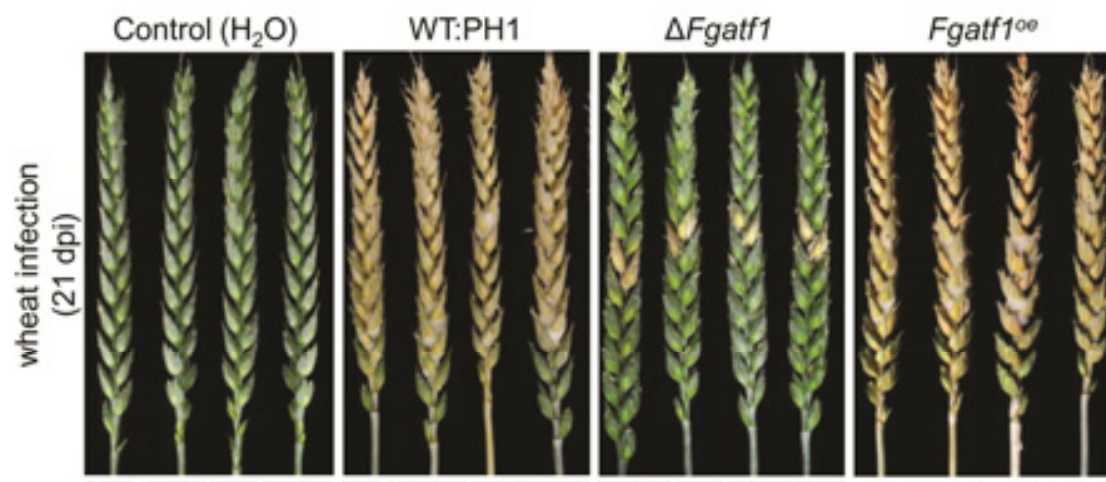

Fgatf10e:: $\triangle F g O S-2$

$\triangle \mathrm{FgOS}-2$
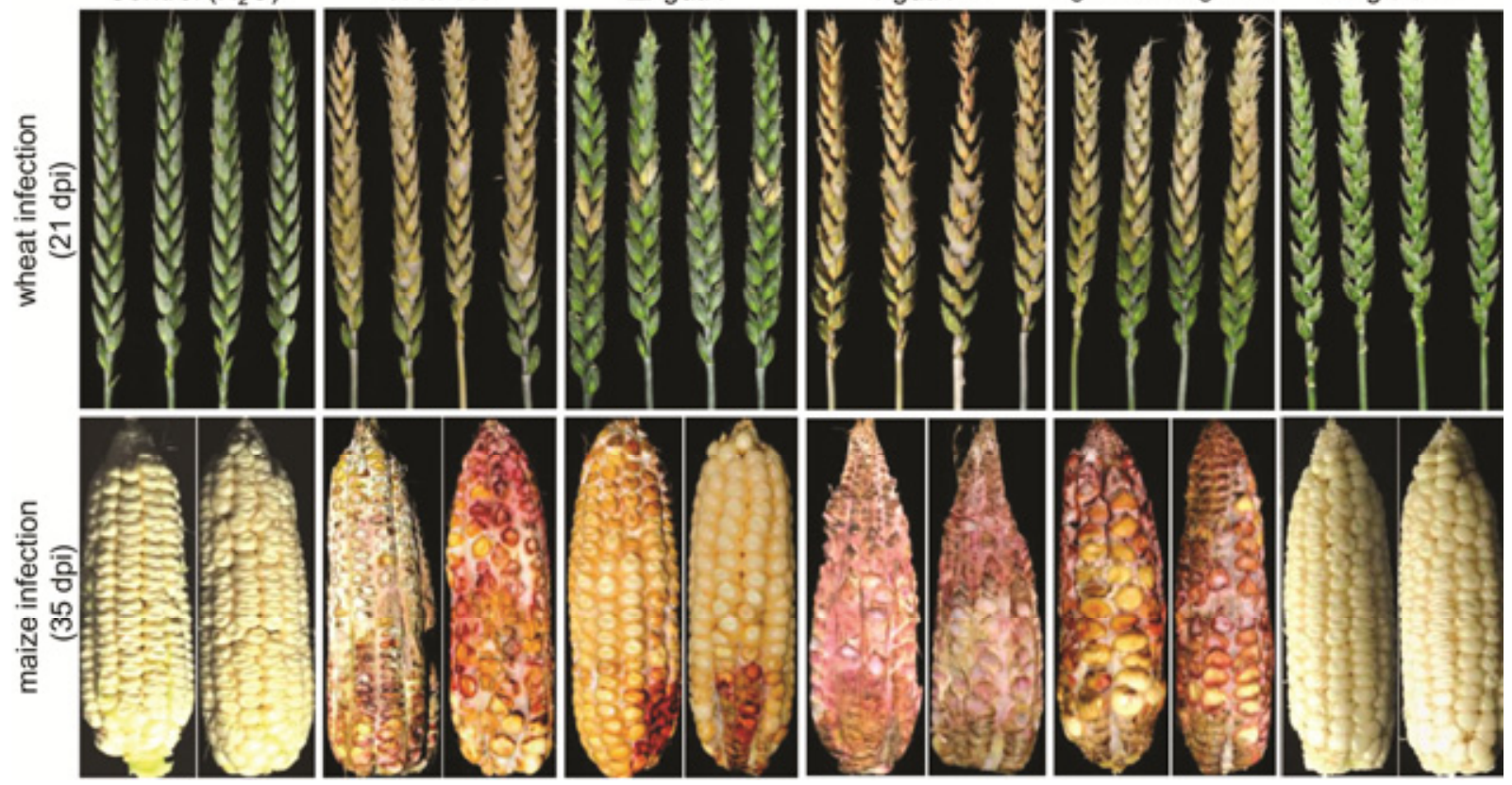

Fig. 5. Pathogenicity assay on wheat and maize. Wheat heads (upper row) and maize cobs (lower row) were inoculated with conidia of the wild type (WT:PH1) and the $\Delta F g a t f 1, F g a t f 1^{\circ e}, \Delta F g O S$-2, and $F g a t f 1^{\circ e}:: \Delta F g O S-2$ mutants and incubated for 35 (maize) and 21 (wheat) days. Inoculation with water served as a control. The $\Delta$ Fgatfl mutant strain was reduced in virulence toward both hosts. On wheat, the infection of wheat stopped in the inoculated spikelet. The wild-type and the Fgatf $1^{\mathrm{oe}}$ and $F g a t f 1^{\mathrm{oe}}:: \Delta F g O S-2$ strains caused symptoms typical for maize cob rot and Fusarium head blight disease. Maize infections were repeated 6 times and the wheat infections performed 30 times. 
$\Delta$ Fgatfl mutant was unable to bridge the transition zone (rachis node) between the inoculated spikelet and the transmitting tissue. This finding was verified by histological observations on longitudinal sections of single spikelets and entire spikes inoculated with a $\Delta$ Fgatfl strain and the wild type, respectively, that were both transformed with vector pII99::dsRed (Ilgen et al. 2009) that facilitates the expression of the red-fluorescent protein dsRed (Fig. 6). After 7 dpi, the $\Delta$ Fgatfl-dsRed strain colonized the inoculated spikelet but growth toward the rachis node was attenuated. At this timepoint, the wild type already passed the rachis node at the bottom of the inoculated spikelets and grew inside the adjacent one (Fig. 6). Within $21 \mathrm{dpi}$, the wild type colonized the complete spike. Hyphal growth can be seen in each spikelet below and above the point-inoculated one. Growth of the $\Delta F$ gatfl mutants in contrast ceased in the inoculated spikelet (Fig. 6).

The $\Delta F$ gatfl were also reduced in virulence toward maize. All maize cobs inoculated with the Fgatfl deletion strains were only superficially colonized. The wild type colonized the entire maize cob within the 35 dpi (Fig. 5).

Constitutive expression of Fgatfl increased the virulence. On susceptible 'Nandu' wheat, infection proceeded faster compared with the wild type (two independent mutants were tested) (Fig.
5). This accelerated infection became more obvious on the more resistant wheat 'Amaretto'. The average infection of the Fgatfl $1^{\text {oe }}$ mutants proceeded more quickly and spread further compared with the wild-type infection (Supplementary Fig. S11A). The same applied to the infection on the close relative to cereal grain species, Brachypodium distachyon. When inoculated with the wild type, the infection usually stopped in the point-inoculated spikelet. However, the average infection of the Fgatfl-overexpressing mutant proceeded beyond the inoculated spikelet. Intriguingly, the constitutive expression of Fgatfl partially complements the reduced pathogenicity toward wheat and maize resulting from the deletion of $\mathrm{FgOS}$-2 (three independent mutants were tested) (Fig. 5). The analysis of DON production in the Fgatf ${ }^{\text {oe }:: \Delta F g O S-2}$ mutants revealed a massive increase in DON production compared with the $\triangle F g O S-2$ mutants (discussed below) during wheat head colonization. This increase might contribute to the reconstitution of pathogenicity.

Taken together, our results suggest that FgAtf1 plays a pivotal role in proper pathogenic development of $F$. graminearum on different hosts. Furthermore, we can now hypothesize that FgOS-2 regulates DON biosynthesis not only through FgAtf1 but also through at least one more transcriptional regulator that is as yet unknown.
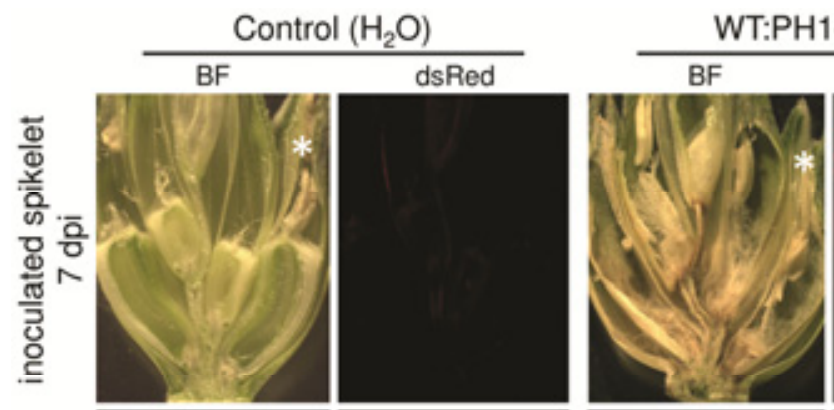

\section{1-dsRed}
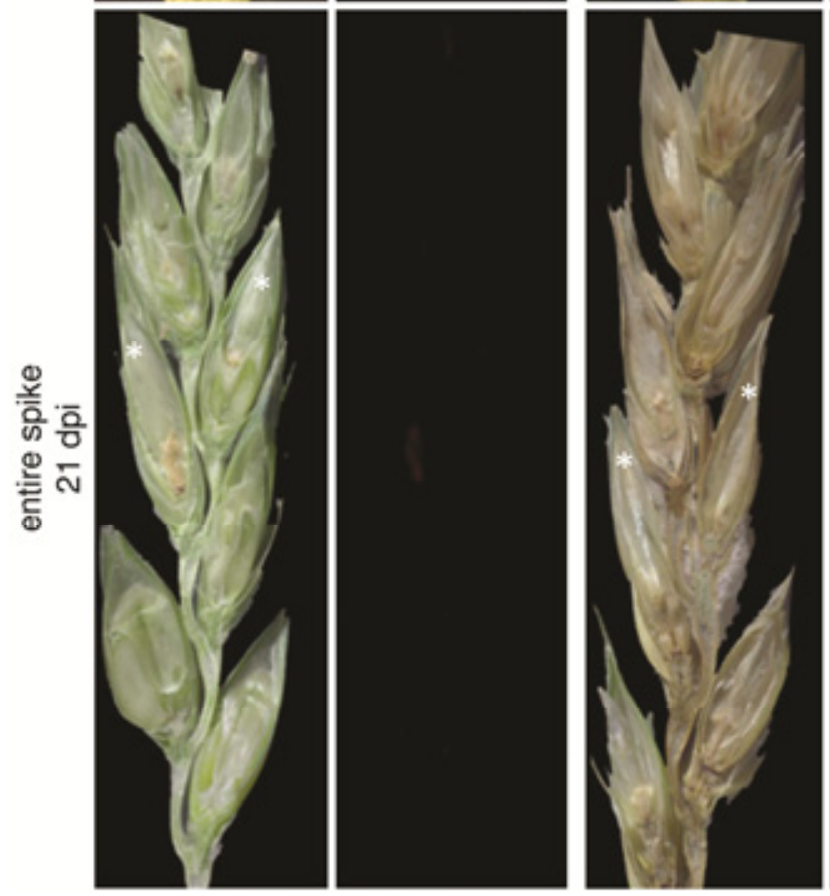
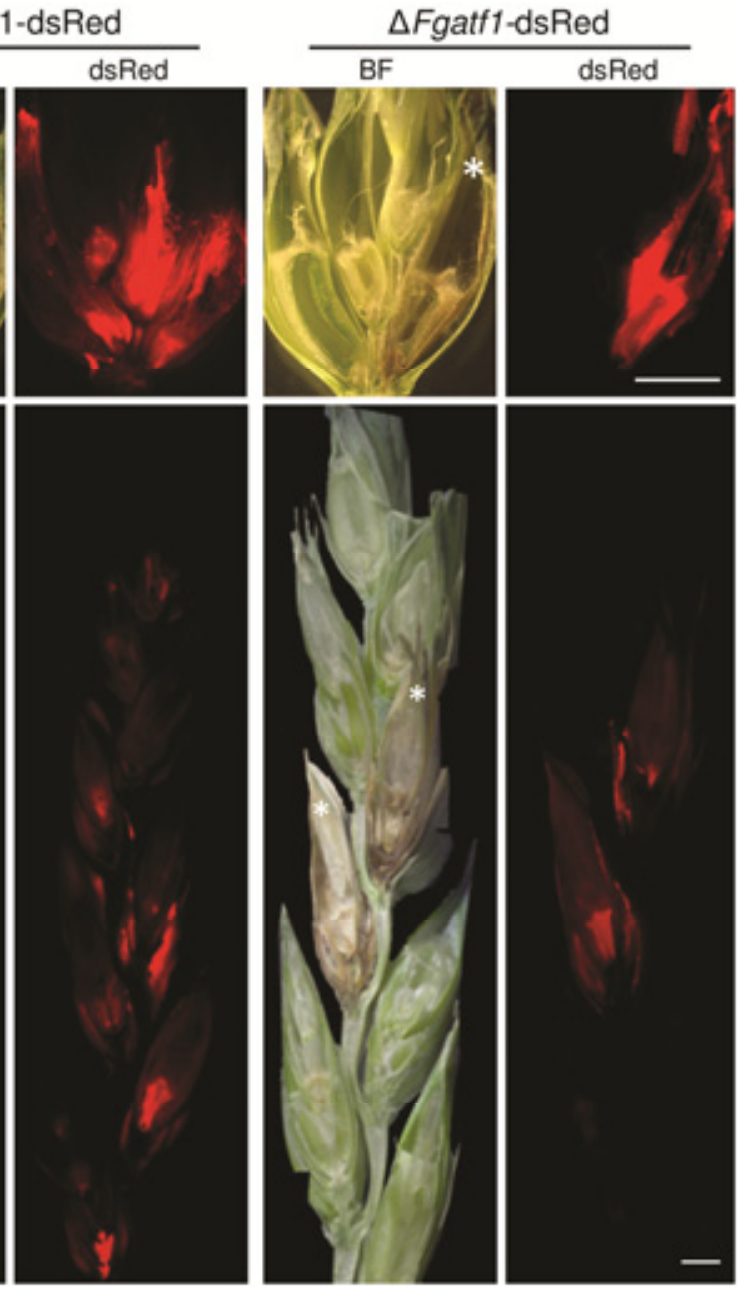

Fig. 6. Infection assay. Cross-sections of inoculated wheat spikelets (upper row) and entire spikes (lower row). Spikelets were inoculated with strains that were derived from the wild type (WT:PH1) and the $\Delta F$ gatfl-strain. They express dsRed constitutively in the cytosol. Inoculation with water served as a control. Inoculated spikelets: overview cross-section, including the inoculated spikelet and the rachis node at 7 days postinoculation (dpi). Inoculation points are indicated with white asterisks. The $\Delta F$ gatfl mutant infection was attenuated compared with the wild-type-derived mutant. Bright-field microscopy revealed disease symptoms in the inoculated spikelet but not in the adjacent one. The wild-type-derived strain completely colonized the inoculated and the adjacent spikelet. Entire spikes (composite picture): infection of the $\Delta F g a t f 1$ mutant stopped in the inoculated spikelet, while the wild-type derived strain successfully colonized the complete spike at $21 \mathrm{dpi}$. Scale bar $=1 \mathrm{~mm}$. 


\section{F. graminearum Atf1 orchestrates secondary metabolite production.}

The ability to produce the trichothecene mycotoxin DON and the pathogenic potential toward wheat correlate (Maier et al. 2006; Proctor et al. 1995). Mutants deficient in the gene encoding a trichodiene synthase are unable to grow through the rachis node. Deletion of $\mathrm{FgOS}$-2 leads to a reduced DON production in planta and, thus, to a reduced virulence (Nguyen et al. 2012). To address the question of whether FgAtf1 regulates DON-production, highly sensitive enzyme-linked immunosorbent assay (ELISA) measurements were conducted using infected wheat spikelets and samples obtained from liquid toxin-induction cultures. The assay was conducted using the

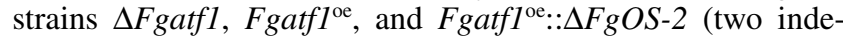
pendent mutants each). The results are summarized in Supplementary Table S2. Interestingly, in planta, the $\Delta$ Fgatf1 DON level was between the wild-type and $\Delta F g O S$-2 DON levels. The Fgatfl deletion strains produced approximately 3 times less DON than the wild type and approximately 20 times more than the $\Delta F g O S-2$ mutants 7 dpi (Fig. 7) (Nguyen et al. 2012). As mentioned above, constitutive expression of Fgatfl increased DON production in planta and led to a higher aggressiveness of $F$. graminearum toward different hosts. This upregulation even overrules the strong decrease in DON production in the mutants in which $\triangle F g O S-2$ is deleted. This finding suggests that FgAtf1 contributes to the regulation of DON biosynthesis during plant infection. Intriguingly, under in vitro conditions, the $\Delta F$ gatfl mutants produced much more DON than the wild type (and the $\Delta F g O S-2$ mutant). Compared with the wild type, the $\Delta$ Fgatfl mutant produced approximately 3.5 and 1.7 times more DON after 1 and $3 \mathrm{dpi}$, respectively. All measurements are normalized to the amount of fungal material in the sample using quantitative (q)PCR (Voigt et al. 2007). Expression of four genes involved in DON-production (tri4, encoding a P450 monooxygenase; tri5, trichodiene synthase; and tri6 and tri10,

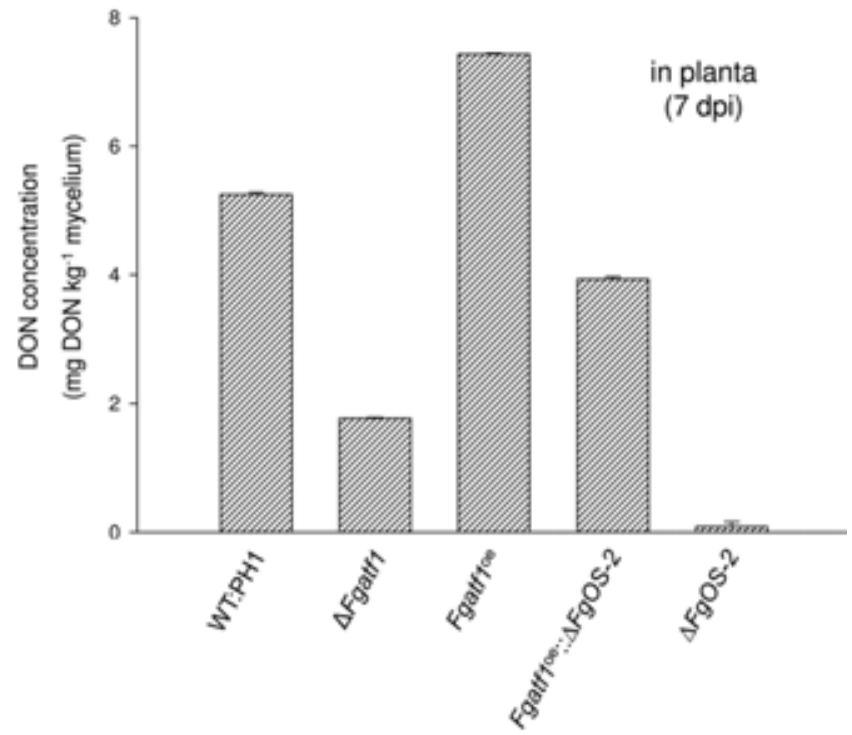

Fig. 7. Deoxynivalenol (DON) concentrations in wheat heads infected with the wild type (WT:PH1), the Fgatf1 mutants ( $\Delta F$ gatfl, Fgatf1 ${ }^{\mathrm{oe}}$, and $\left.F g a t f 1^{\mathrm{oe}}:: \Delta F g O S-2\right)$, and the $\triangle F g O S-2$ mutant. The DON concentration was determined by enzyme-linked immunosorbent assay at 7 days postinoculation (dpi). The DON contents were lower in the Fgatf1 deletion strains but higher in Fgatf ${ }^{\mathrm{oe}}$ mutants compared with the wild type. As described previously (Nguyen et al. 2012), the $F g O S-2$ deletion strains produce less DON during wheat infection. This lack in DON production is partially complemented by the overexpression of Fgatf1. Toxin measurements were repeated twice with four replicates each. Error bars indicate the standard deviation. both transcription regulators) was regulated accordingly (Supplementary Table S3). This substantiates our previous finding that FgOS-2, in concert with the downstream transcriptional regulator FgAtf1, is the central trigger for growth-conditiondependent DON production. Furthermore, our results indicate a dominant inhibitory effect of FgAtfl on the production of DON under in vitro conditions. Whether this is an effect of direct regulation or due to pleiotropic effects demands further investigation.

A second important mycotoxin, ZEA, accumulates in infected grains and is also produced in axenic culture (Gaffoor et al. 2005). After $7 \mathrm{dpi}$ on wheat spikes, the $\Delta$ Fgatfl mutant synthesized significantly less ZEA compared with the wild type. As already observed for the $\triangle F g O S-2$ mutant (Nguyen et al. 2012), the transcript level of the putative regulatory transcription factor (zeb2) was higher in the Fgatfl deletion mutants compared with the wild type at 7 dpi. Expression of genes encoding an isoamyl alcohol oxidase (zebl) and a nonreducing polyketide synthase (zeal) was reduced in planta compared with the wild type. Under in vitro induction conditions, there is no difference in ZEA production (and the regulation of gene expression) between $\Delta F$ gatfl and the wild type.

A third prominent secondary metabolite of $F$. graminearum is the red pigment aurofusarin. Regulation of aurofusarin production is regulated in an FgOS-2-dependent manner (Nguyen et al. 2012). This regulation partially takes place via FgAtf1signaling, because pigment production and expression of genes involved in aurofusarin biosynthesis ( gipl, encoding a putative laccase; gip2, transcription factor; and $p k s 12$, polyketide synthase) were upregulated in $\Delta F g a t f l$ mutants. This led to an increased red coloration of the mutant colonies (Supplementary Fig. S12).

Taken together, these results underline the importance of the FgOS-2/FgAtf1-signaling cascade in the regulation of secondary metabolite production.

\section{F. graminearum Atf1 acts as a repressor of catalase gene expression and activity under oxidative stress conditions.}

We assume that the global ROS metabolism in $F$. graminearum is modulated by the FgOS-2/FgAtf1 signaling machinery mainly by regulation of catalase gene expression and activity. $\mathrm{FgOS}$-2 deficiency leads to a massive induction of catalase gene expression and enzyme activity after exposure to $\mathrm{H}_{2} \mathrm{O}_{2}$ (Nguyen et al. 2012). In this regard, we tested gene expression and enzyme activity of four putative catalase genes under different conditions (osmotic, oxidative stress, and during wheat infection) in the different Fgatfl mutants (two independent $\triangle F$ gatfl, $F g a t f 1^{\mathrm{oe}}$, and $F g a t f 1^{\mathrm{o}}:: \Delta F g O S-2$ mutants in each experiment) and the $\triangle F g O S-2$ mutants (two mutants tested) (Fig. 8; Supplementary Table S4A to C). Intriguingly, gene expression of all catalase genes was drastically elevated under oxidative stress conditions in the $\Delta F$ gatfl mutants compared with the wild type (Fig. 8B). A catalase activity assay confirmed that oxidative stress also increased the overall activity of catalases in the $\Delta$ Fgatfl mutants compared with the reference condition (only CM), and the wild type (stressed with $\mathrm{H}_{2} \mathrm{O}_{2}$ ) (Fig. 8D). Deletion of Fgatfl did not affect the expression of catalases 1 and 3 (cat 1 and cat 3 ) during wheat infection. However, in both mutants with constitutive expression of Fgatfl (Fgatf ${ }^{\text {oe }}$ and $F g a t f 1^{\mathrm{oe}}:: \Delta F g O S$-2), expression of these two catalase genes was much lower than in the wild type but approximately six and five times higher, respectively, compared with $\mathrm{FgOS}$-2 deletion strains (Fig. 8A). Moreover, expression level of cat 2.1 and cat 2.2 was elevated in the Fgatf $1^{\text {oe }}$ mutants compared with the $\triangle F g O S$-2 mutant and the wild type during wheat infection. As mentioned earlier, constitutive expression 
of Fgatfl increases virulence and complements the corresponding phenotype of $\Delta F g O S$ - 2 mutants. It seems feasible that the elevated expression of two catalase genes contributes to an enhanced immunity against a putative hypersensitive plant defense reaction. Exposure to osmotic stress evoked an increase in transcript level of the catl gene in the $\Delta$ Fgatfl mutants: it was upregulated approximately three times compared with the wild type (Fig. 8C). However, this upregulation did not lead to an increase in overall catalase activity (Fig. 8D). Interestingly, constitutive expression of Fgatfl in the $\Delta \mathrm{FgOS}-2$ mutant background led to a significant increase in cat 1 expression compared with $\triangle F g O S-2$ mutants. This elevation might contribute to the partial reversion of the hypersensitivity phenotype of the $\Delta F g O S-2$ mutant toward osmotic stress. The expression levels of the other catalases (cat2.1, cat2.2, and cat3) remained more or less unchanged compared with the wild type. Taking these results, we assigned to FgAtf1 a function as repressor of catalase gene expression in response to oxidative stress. Whether this is a direct regulation or not and which protein, in turn, acts as a positive regulator of catalase expression remains open (Fig. 9).

\section{DISCUSSION}

ROS are unavoidable byproducts in all aerobic organisms. Along with their often harmful effects on cell walls, membranes, lipids, and DNA (Finkel 2003), ROS are also recognized as important signaling molecules in the host-pathogen interaction
(Apel and Hirt 2004; Heller and Tudzynski 2011). In a previous study, we characterized the stress-activated protein kinase FgOS-2 as a central regulator in the life cycle of $F$. graminearum (Nguyen et al. 2012). Our results led to the conclusion that many of the observed phenotypes were related to a disturbed ROS equilibrium. Well-balanced ROS equilibria are crucial for proper development in plants (Foreman et al. 2003), mammals (Ježek and Hlavatá 2005; Thannickal and Fanburg 2000), and fungi (Scott and Eaton 2008). In the present study, we analyzed the putative downstream target of FgOS-2, the basic leucine zipper transcription factor FgAtf1, in regard to its importance for pathogenicity, secondary metabolite production, and sexual reproduction. In some aspects, we found additional evidence for our hypothesis of a causal connection between a disruption of the ROS homeostasis and the observed phenotypes. However, because some phenotypes observed in the $\Delta F$ gatfl mutants only partially resemble the phenotypes observed upon $\mathrm{FgOS}$-2 deletion, we postulated additional, asyet-unknown downstream targets of FgOS-2 (Fig. 9). Interestingly, the constitutive overexpression of Fgatfl increases virulence and partially complements deficiencies in this and other regards in $\triangle F g O S$-2 mutants. Whether this effect is solely explainable by the relatively higher abundance of Fgatfl transcript in these mutants or whether it benefits from an FgOS-2-independent posttranslational activation remains to be tested. Furthermore, we demonstrated that FgAtf1 constitutively localizes to the nucleus regardless of the genetic background (i.e., $\Delta F g O S$-2) or the growth conditions.
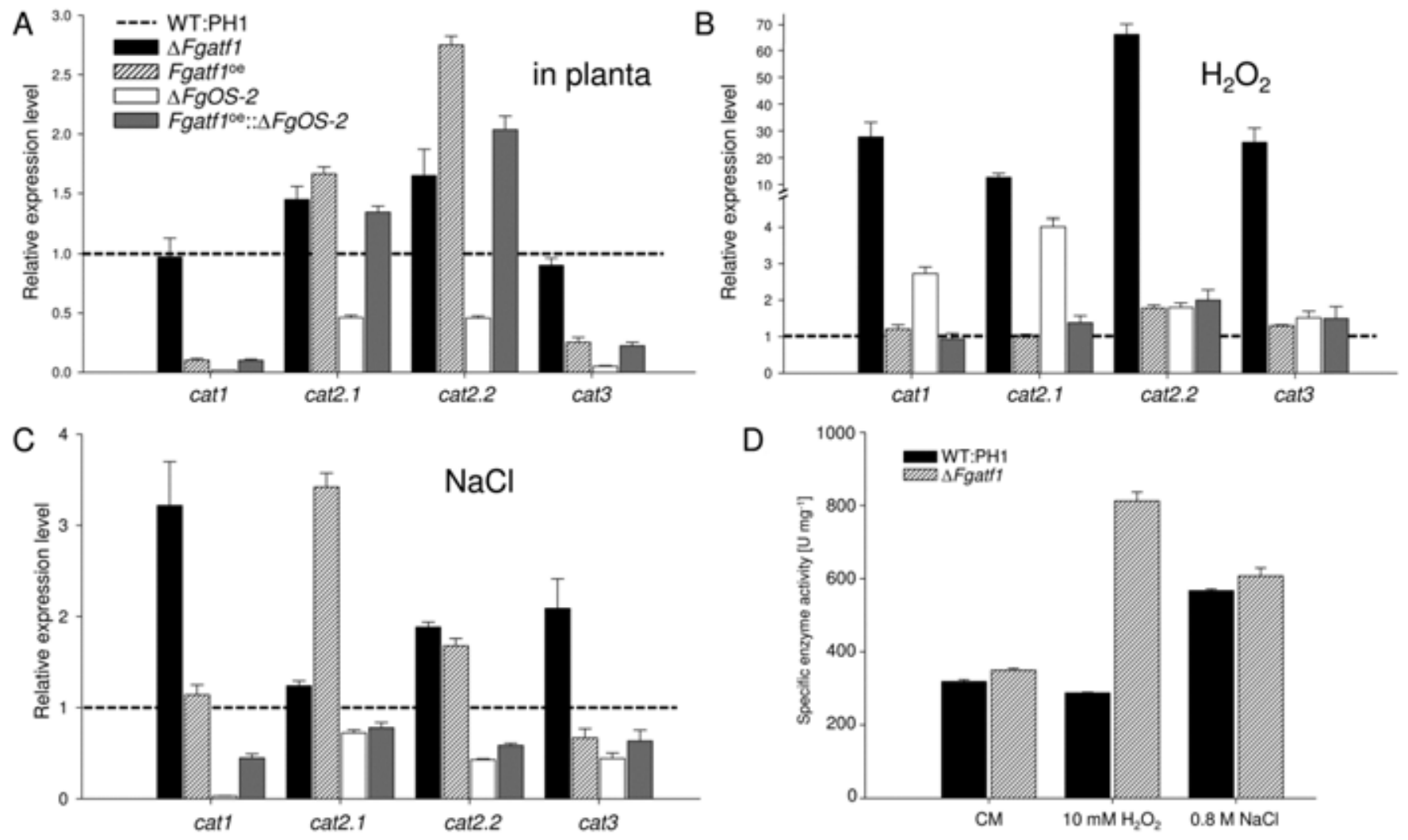

Fig. 8. Catalase expression and activity assay. A to C, Quantitative reverse-transcription-polymerase chain reaction (qRT-PCR) using cDNA obtained from A, inoculated wheat spikelets (7 days postinoculation [dpi]; $\mathbf{B}, \mathrm{H}_{2} \mathrm{O}_{2}$-supplemented (10 mM); and C, NaCl-supplemented samples ( $\left.0.8 \mathrm{M}\right)$. Expression of four different, putative catalase genes was assayed in the wild type (WT:PH1), the Fgatf1 mutants $\left(\Delta F\right.$ gatf1, Fgatf $1^{\text {oe }}$, and $\left.F g a t f 1^{\circ}:: \Delta F g O S-2\right)$, and the $\triangle F g O S$-2 mutant. The wild-type expression level was set to 1 . Catalase expression in the $\Delta$ Fgatfl mutant strain was not drastically changed during the infection of wheat. Except catalase 1, which was upregulated approximately three times, all catalases were only slightly upregulated in the $\Delta F g a t f 1$ mutant in response to osmotic stress $(\mathrm{NaCl})$. In the presence of oxidative stress $\left(\mathrm{H}_{2} \mathrm{O}_{2}\right)$, all catalases were drastically upregulated in the $\Delta F g a t f 1$ mutant compared with the wild type. qRT-PCR was performed in triplicate. D, Catalase activity measurement using native protein extract from the wild type and the $\Delta$ Fgatfl mutant strains. Cultures were supplemented with $0.8 \mathrm{M} \mathrm{NaCl}$ and $10 \mathrm{mM} \mathrm{H}_{2} \mathrm{O}_{2}$, respectively. Under oxidative stress condition, catalase activity was massively upregulated in the $\Delta$ Fgatfl mutant compared with the wild type. Error bars indicate the standard deviation. 


\section{F. graminearum Atf1 plays an ambivalent role in stress response.}

We drew a connection between fungal growth, the alteration of ROS level in response to environmental stresses, and a regulation of catalases in F. graminearum (Nguyen et al. 2012). A pivotal role in this response regulation is assigned to FgAtf1. Here, we show that the contribution of FgAtf1 varies depending on the type of stress and suggest that FgAtf1 is less important for mycelia growth under salt stress conditions compared with FgOS-2. Thus, it is feasible that FgOS-2 directly regulates the osmotic stress response or that it does not activate FgAtf1 exclusively but also other transcription factors. In Saccharomyces cerevisiae, the Hog1 MAP kinase regulates at least four transcription factors: namely, Msn2, Msn4, Hot1, and Sko1 (Hohmann 2002). Growth of Fgatfl deletion strains was still possible on medium containing $0.8 \mathrm{M} \mathrm{NaCl}$. At this concentration, mycelia growth of the $\Delta F g O S-2$ completely ceased, most likely because the mutant produces plenty of ROS and is no longer able to induce catalase gene expression in order to detoxify them (Nguyen et al. 2012). Unlike the $F g O S$-2 deletion strains, $\Delta F$ gatfl mutants did not evoke an increase in ROS accumulation when exposed to osmotic stress. Furthermore, catalase gene expression and activity were not much elevated in $\Delta$ Fgatfl mutants under osmotic stress compared with the wild type (Fig. 8C). In this regard, however, it is noteworthy that we observed an unusual germination pattern in $\Delta F$ gatfl mutants under osmotic stress conditions. $\Delta F$ gatfl germ tubes did not swell, were not polynuclear, and did not stop growing after a short period of growth; however, they emerged only at the apex of the conidium, as they did in the $\triangle F g O S$ - 2 mutants (Nguyen et al. 2012). In this context, the regulation pattern of a putative spore-specific catalase (Navarro et al. 1996; Paris et al. 2003) (cat1 in $F$. graminearum) is remarkable. In $\Delta F g O S$ - 2 mutants, the transcript level of this catalase is very low (Nguyen et al. 2012), whereas it is increased three times in the $\Delta F$ gatfl under osmotic stress conditions compared with the wild type. This upregulation might contribute to a higher stress tolerance of conidia on osmotic stress medium. Consequently, upregulation of all four catalase genes in the Fgatf $1^{\mathrm{oe}}:: \Delta F g O S$-2 mutants may contribute to the full restoration of growth on mild osmotic stress medium (Fig. 1B) by the restoration of ROS detoxification capabilities. BiFC experiments demonstrated that FgOS-2 and FgAtf1 physically interact under salt stress conditions (Fig. $3)$. This interaction is also documented for Aspergillus nidulans (Lara-Rojas et al. 2011). However, in this fungus, the interaction occurs in response to oxidative stress. In $B$. cinerea, it is feasible that the physical interaction takes place under osmotic stress condition, because Heller and associates (2012) localized the $B$. cinerea HOG1 homolog BcSak1 in the nucleus after exposure to osmotic stress. In F. graminearum, Atf1 permanently resides in the nucleus whereas FgOS-2 translocates into the nucleus upon osmotic stress, presumably after getting phosphorylated (Zheng et al. 2012). Intriguingly, FgAtf1 localization is not affected by osmotic or oxidative stress and the presence or absence of FgOS-2. Hence, it is feasible that the physical interaction of both proteins inside the nucleus is necessary for the proper response to osmotic stress. This would explain the sensitivity of both the $\Delta F g O S$ - 2 and the $\Delta F g a t f l$ mutant toward osmotic stress and would fit the situation in $S$. pombe, where the stress-induced gene regulation relies on the interaction of the OS-2 ortholog Sty1 and Atf1 (Reiter et al. 2008).

In regard to oxidative stress response, FgAtf1 seem to be the major regulator. $\Delta F$ gatfl mutants grew much better than the wild type (and $\triangle F g O S$-2) on medium containing up to $20 \mathrm{mM}$ $\mathrm{H}_{2} \mathrm{O}_{2}$ (Fig. 2). This increase in oxidative stress resistance is likely due to the drastic upregulation of catalase gene expression and catalase activity under oxidative stress conditions. This finding highlights again the idea that functions of the Atf1 homologs within the polyphyletic group of necrotrophic plant pathogens evolved differently, because deletion of the $M$. oryzae Atf1 ortholog (Moatf1) leads to hypersensitivity against oxidative stress (Guo et al. 2010). The authors connect this phenotype to the mutant's disability to detoxify ROS. The $B$. cinerea Atf1 ortholog is reported to act as a regulator of catalase B gene expression upon exposure to oxidative stress (Temme et al.

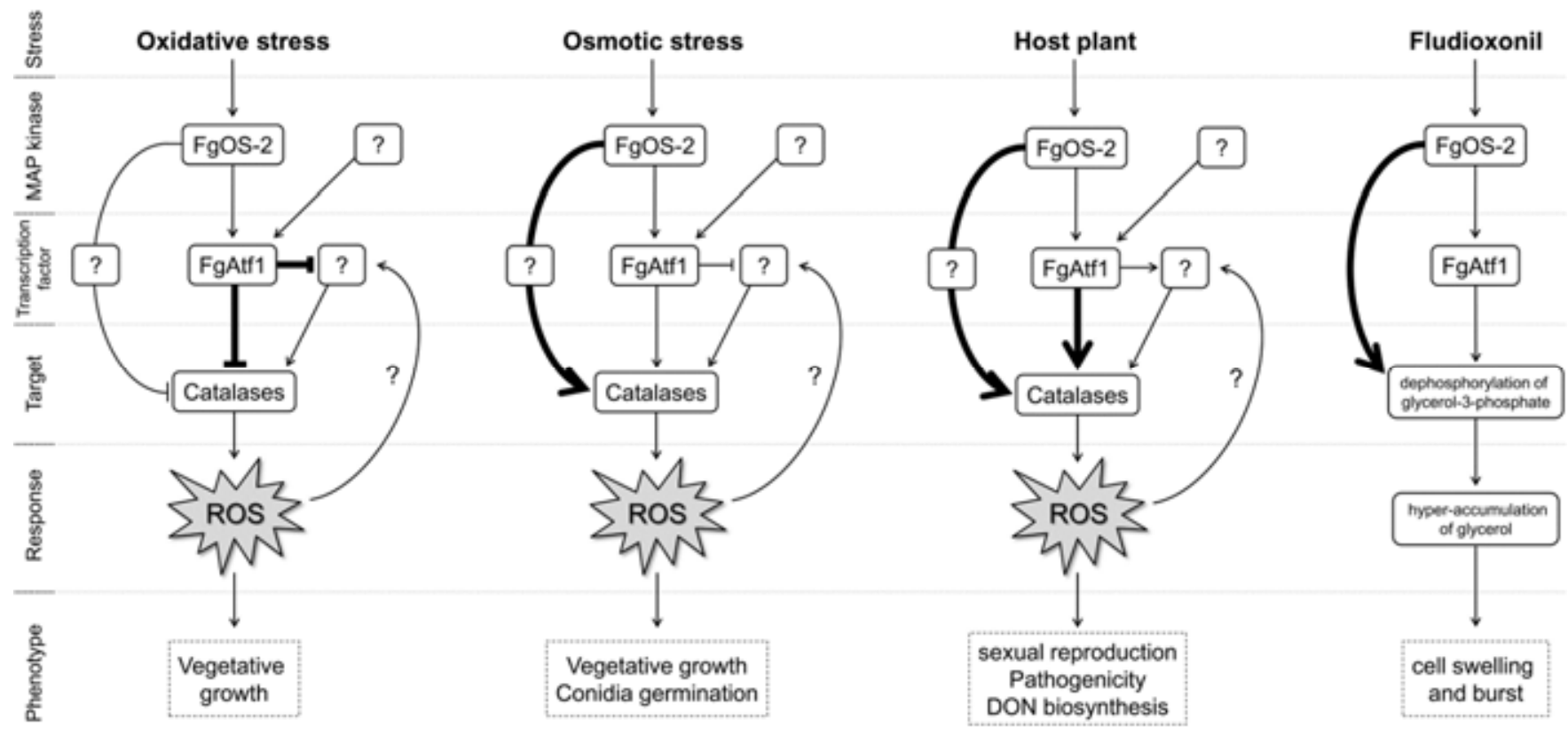

Fig. 9. Proposed model. How FgOS-2 and FgAtf1 influence numerous physiological functions like vegetative growth, sexual reproduction, germination, and pathogenicity in Fusarium graminearum. FgAtf1 is activated by FgOS-2 and environmental cues such as a host plant or osmotic or oxidative stress conditions. Among others, FgAtf1 contributes to reactive oxygen species (ROS) production and degradation, presumably by regulating ROS metabolic enzymes such as catalases. In this regulatory system, we have to assume a substantial contribution by additional, as-yet-unknown factors. Heavy arrows indicate the relatively higher importance of a particular connection under certain conditions. 
2012). Catalase B expression is diminished as a result of the deletion of Bcatfl. Nonetheless, $\Delta b c a t f 1$ mutants show no increased sensitivity toward oxidative stress mediated by $\mathrm{H}_{2} \mathrm{O}_{2}$. Thus, in regard to viability under oxidative stress conditions, the importance of Atf1 varies in necrotrophic fungi from dispensable (B. cinerea) over essential $(M$. oryzae) to inhibitory (F. graminearum). In $F$. graminearum, both FgOS-2 and FgAtf1 are not essential for vegetative growth under oxidative stress conditions and might even act as a repressor of catalase gene expression. Contrary to expectations, constitutive expression of Fgatf1 neither increases nor decreases sensitivity toward oxidative or osmotic stress but, interestingly, caused an increased aggressiveness toward several host plants (Fig. 5) (discussed below). The Fgatfl deletion mutants were not resistant against fludioxonil as was observed in $\mathrm{FgOS}$-2 deletion strains. Application of fludioxonil leads to an upregulation of a glycerol-3phosphate phosphatase (Nguyen et al. 2012), which dephosphorylates glycerol-3-phosphate (Albertyn et al. 1994; Pillonel and Meyer 1997). In consequence, glycerol accumulates and the cells swell and frequently burst. The fact that Fgatfl deletion mutants are as sensitive to fludioxonil as the wild type underscores that FgOS-2 possesses unique functions that are independent of FgAtf1. In Neurospora crassa, Atf1 and OS-2 share some functions in response to fludioxonil, although $\Delta$ Fgatfl strains were as susceptible to this fungicide as the wild type (Yamashita et al. 2008).

\section{Secondary metabolite production depends on functional FgAtf1.}

Proper regulation of secondary metabolite production depends on the FgOS-2 signaling cascade and culture conditions. Production of prominent secondary metabolites such as DON, ZEA, and aurofusarin is regulated by FgOS-2 in a growth-conditiondependent manner (Nguyen et al. 2012). Although the $\Delta F g O S-2$ mutants produced more DON under in vitro induction conditions, biosynthesis was reduced during wheat infection. Aurofusarin synthesis, however, was deregulated and occurred also (unusually) during wheat head infection. Deletion of Fgatfl also elevates aurofusarin production. Aurofusarin exhibits antimicrobial potential and inhibits fungal growth (Malz et al. 2005; Medentsev et al. 1993). Thus, it is feasible that, due to an upregulation of aurofusarin biosynthesis, vegetative growth of the $\Delta F$ gatfl strains is reduced. Atf1 was assigned a function in the regulation of secondary metabolism before. Deletion of $\mathrm{B}$. cinerea Atf1 resulted in an upregulation of phytotoxin production (Temme et al. 2012). Also, FgAtf1 is of major importance for the regulation of mycotoxins and, again, the regulation pattern turned out to depend on the transcript level of Fgatfl and the culture conditions. Absence of Fgatfl led to a drastic increase in DON production under in vitro conditions compared with the wild type but a strong reduction during wheat infection. Intriguingly, constitutive expression of Fgatfl resulted in an increased DON production during wheat infection. As shown by Ponts and associates (2006), DON production in vitro is induced by $\mathrm{H}_{2} \mathrm{O}_{2}$. Whether this is the main inducer of DON biosynthesis during wheat infection is still under investigation. During wheat infection, catalase gene expression is much higher in the $\Delta F g$ atfl mutants compared with the $\Delta F g O S$ - 2 mutants but not much changed compared with the wild type (Fig. 8A). Hence, ROS might not accumulate in tissues infected with the $\Delta$ Fgatfl mutants. This might partially explain why DON production is much higher in the $\Delta F$ gatfl mutants compared with the $\Delta F g O S-2$ mutants, although it is still lower compared with the wild type. These results underline the idea that FgOS-2 regulates DON production not only by the regulation of FgAtf1 but also through other, as-yet-unknown factors. Also, proper ZEA production relies on FgAtf1. In vitro, ZEA levels are nearly wild type like, whereas ZEA production is diminished in planta in the $\Delta F$ gatfl mutants. We observed the same regulation pattern in $\Delta F g O S$-2 mutants (Nguyen et al. 2012). Secondary metabolite production is generally influenced by different culture conditions (Calvo et al. 2002; Keller and Hohn 1997; Kim et al. 2005; Linnemannstöns et al. 2002). ZEA biosynthesis is mainly induced by grain-specific factors (Gaffoor et al. 2005). Hence, as already noted for DON production, FgAtf1 interferes specifically with plant-derived cues. In vitro, however, the regulatory pathways are fundamentally different. This underscores the belief that it is inevitable to determine the conditions that induce secondary metabolite production during wheat infection.

\section{Balanced Fgatf1 transcript levels are a prerequisite for sexual reproduction.}

$F g O S$-2 deficiency leads to a complete loss of sexual reproduction (Nguyen et al. 2012). Interestingly, deletion of atfl in $F$. graminearum also negatively influenced formation of reproductive structures. Development of perithecia was delayed in $\Delta$ Fgatfl mutants compared with the wild type on carrot agar and on detached wheat nodes but ascospores forcibly discharged from those perithecia were viable. Deletion of the $N$. crassa atfl ortholog, asl-1, in contrast, led to nonfunctional ascospores (Colot et al. 2006). The inability of the $\triangle F g O S-2$ mutants to produce perithecia and ascospores can be restored by a constitutive expression of Fgatfl. This attributes to FgAtf1 a prominent role in the regulation of sexual reproduction. Because the deletion of Fgatfl does not lead to full sterility, other factors may also interfere. Perithecia formation might be regulated through distinct ROS levels. There are examples from other fungi such as A. nidulans (Lara-Ortiz et al. 2003), Podospora anserina (Malagnac et al. 2004), and N. crassa (CanoDomínguez et al. 2008), in which disturbance in ROS balance by deletion of NADPH oxidases lead to disability to reproduce sexually. Hence, it is feasible that the misregulation of catalases in $F$. graminearum interferes with mating processes and, thereby, interrupts sexual development.

\section{Virulence of $\boldsymbol{F}$. graminearum relies on Fgatf1 transcript level.}

Our results suggest a close relationship between Fgatfl transcript level and pathogenicity toward wheat and maize. Furthermore, Fgatfl expression is regulated by FgOS-2. In FgOS-2 deletion mutants, Fgatfl transcription is very low and so is virulence toward both hosts (Nguyen et al. 2012). In the present study we showed that deletion of Fgatfl from the genome led to a severe reduction in virulence against wheat and maize. In contrast, an overexpression of Fgatfl results in a greater aggressiveness against these hosts (and Brachypodium distachyon). Strikingly, constitutive expression of Fgatfl partially restored virulence of $\mathrm{FgOS}$-2 deletion strains (Fig. 5). This strongly suggests that FgAtf1 is a superior regulator of virulence in $F$. graminearum. However, the question remains open as to whether the high abundance of Fgatfl transcript is already sufficient to increase the virulence or FgAtf1 function requires a posttranslational modification (i.e., through phosphorylation). Atf1 orthologs are described to be activated by phosphorylation through an upstream (HOG1) MAP kinase (Moye-Rowley

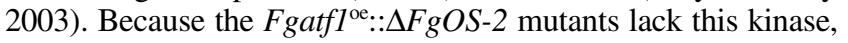
we have to assume that FgAtf1 either gets autophosphorylated or is the target of a different, as-yet-unknown MAP kinase that translocates to the nucleus under certain conditions. The Sko1 of Saccharomyces cerevisiae and Atf1 of Schizosaccharomyces pombe are known to be negatively regulated by the cAMP-dependent protein kinase A and positively regulated by a MAP kinase (Davidson et al. 2004; Pascual-Ahuir et al. 2001). In the 
case of $S$. pombe, this kinase is PMK1 (Takada et al. 2007), which is well described in $F$. graminearum (Jenczmionka and Schäfer 2005; Jenczmionka et al. 2003; Urban et al. 2003) but, thus far, was not involved in SAPK signaling cascades, although there are overlapping phenotypes in the deletion mutants of FgOS-2 and Gpmk1, especially regarding sexual reproduction and virulence. Recent results published by Zheng and associates (2012) indicate that - under salt-stress conditions-there is a crosstalk between FgOS-2 and the MAP kinases Gpmk1 and Mgv1. We hypothesize that, upon deletion of FgOS-2, FgAtf1 gets phosphorylated by different MAP kinases. Thus far, to our knowledge, the overruling regulatory function of an Atf1 homolog has not been described. This function has consequences on the transcript level of target genes involved in ROS metabolism. Lamb and associates (2012) found out that, in $N$. crassa, asl- 1 and catalase 1 gene expression was increased under salt stress conditions. In contrast to $F$. graminearum, this increase in catalase expression in $N$. crassa is ASL-1-dependent.

Gene expression analysis on infected wheat spikes revealed a downregulation of all catalase genes in the $\mathrm{FgOS}$-2 deletion strains compared with the wild type and an upregulation in

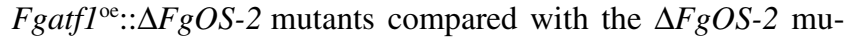
tants. Regulation of balanced ROS levels is important for a proper host-microbe interaction (Scott and Eaton 2008). The drastically reduced virulence of $F g O S$-2-deficient strains and partially restored virulence of the $F g a t f 1^{\mathrm{oe}}:: \Delta F g O S$ - 2 mutant might be due to an altered ROS balance in the interaction between wheat and the mutant strains.

The deletion of the ROS-producing NADPH oxidase 1 in Epichloë festucae leads to a dramatic switch in the life style of this symbiotic fungus: the mutants harmed the viability of their host plants (Tanaka et al. 2006). Deletion of this enzyme in the hemibiotrophic pathogen Claviceps purpurea leads to a decreased virulence toward rye (Giesbert et al. 2008). The importance of Atf1 orthologs for parasitic development varies among the limited number of phytopathogenic fungi in which this gene was functionally characterized thus far. Gene expression of $M$. oryzae atfl (Moatf1) is elevated during germination of conidia and initial penetration of plant surface (Guo et al. 2010). Moatfl deletion mutants are severely attenuated in virulence on rice. The mutant is able to cause small restricted lesions on sprayinoculated rice leaves. Staining experiments using 3,3'diaminobenzidine revealed an enhanced ROS production upon penetration of the plant surface by the mutant, indicating a disturbed ROS balance. Because we could not observe an altered in planta catalase gene expression level upon Fgatfl deletion, misbalance in the ROS level might not be that drastic in the $\Delta$ Fgatfl-wheat interaction. Although the Atf1 homologs of $M$. oryzae and $F$. graminearum obviously both preserved importance for disease development, the $B$. cinerea Atf1 seems to have an even inhibitory effect on virulence, because deletion strains were more aggressive against different hosts (Temme et al. 2012). On inoculated host plants, vigorous hyphal growth is accompanied by a disability to produce conidia. However, we also observed a greater aggressiveness in parasitic growth only upon constitutive expression of Fgatfl. This indicates that, during evolution, Fgatfl orthologs in different organisms gain divergent gene-repressing and -activating capacities, respectively. In $F$. graminearum, the production of DON is prerequisite for successful colonization of wheat. Wheat head infection of mutants deficient (Maier et al. 2006; Proctor et al. 1995) or markedly reduced (Nguyen et al. 2012) in DON production is always restricted to the point-inoculated spikelet. In this study, we observed a significant reduction in DON production by the $\Delta F$ gatfl mutant strains that was, however, still higher than in the $\Delta F g O S-2$ strains. Hence, it is feasible that the reduced virulence toward wheat is due to the reduced DON level. In this regard, it seems likely that the increased DON production observed in the Fgatfl $^{\mathrm{oe}}$ strains as compared with the wild type (Fig. 7) has a positive impact on the pathogenic potential. Gardiner and co-workers (2009b) reported that the deletion of a putative cytochrome P450 monooxygenase and a hypothetical protein with homology to a terpene cyclase leads to an increased DON level and, thus, to an increased virulence toward wheat. Interestingly, both genes are not part of the trigene cluster but their expression is under the control of the positive transcriptional regulator Tri6. Whether or not Fgatf1 is also directly involved in this regulatory machinery remains to be elucidated. The increased production of DON in the Fgatf $^{\text {oe }}$ mutants also elevates the DON accumulation in the Fgatf $1^{\text {oe }:: \Delta F g O S-2}$ strains and, thereby, may contribute to the reversion of the apathogeniciy phenotype of the $F g O S$-2 deletion. Yet, this link between DON production and Fgatfl transcript level does not explain the virulence phenotypes on maize. We observe virulence patterns in the maize infection assay similar to the wheat infection: reduced infection rate in the $\Delta F$ gatfl strain and increased infection in the overexpression mutants (both compared with wild type) (Fig. 5). Although DON is not a pathogenicity factor on maize (Maier et al. 2006), its production is reported to increase the aggressiveness toward this host (Harris et al. 1999). Thus, an increase in DON accumulation might facilitate colonization of maize cobs. In addition, other factors, such as an altered ROS level, may contribute to the increased virulence toward maize.

Altogether, this study highlights the fact that, in F. graminearum, Atf1 acts as one downstream target of FgOS-2. It shares some functions with FgOS-2 but also has important independent features. Most importantly, we found that FgAtf1 comprises physiological functions without $\Delta$ phosphorylation by its putative upstream MAP kinase FgOS-2 (i.e., response to osmotic stress, sexual reproduction, and virulence). In this regard, we have to assume additional, as-yet-unknown factors for activation of FgAtf1. It remains to be elucidated to what extent those factors contribute to the variety of phenotypes we observed. Autophosphorylation is also feasible. To what extent FgOS-2 contributes to the phenotypes in the Fgatfl ${ }^{\text {oe }}$ mutant (in the wild-type background) is subject to ongoing research.

\section{MATERIALS AND METHODS}

\section{Fungal strains and culture conditions.}

All mutants described in this study derived from the $F$. graminearum wild-type strain PH1. Conidiation was induced in liquid wheat medium incubated for 4 days at $28^{\circ} \mathrm{C}$. Sexual reproduction was monitored on carrot agar plates and detached wheat nodes placed on water agar plates (double autoclaved wheat parts with a nodium in the middle). Conidia of the wild type, $\Delta F g O S$-2, and Fgatfl mutant strains ( $\triangle F$ gatfl, Fgatfl ${ }^{\text {oe }}$, and $F g a t f 1^{\mathrm{oe}}:: \Delta F g O S$-2) were inoculated on carrot agar plates and incubated at $28^{\circ} \mathrm{C}$ in the dark. After 3 days, the aerial mycelia were knocked down with $1 \mathrm{ml}$ of sterile $2.5 \%$ Tween 60 solution, using a sterile glass rod. The inoculated carrot agar plates and wheat nodes on water agar plates were further incubated at $18^{\circ} \mathrm{C}$ under near-UV light and white light with a $12-\mathrm{h}$ photoperiod for up to 8 weeks. To observe asci and ascospores in the microscope, the perithecia were excised using a knife, placed on objective slides, and then crushed between the slide and a cover slip. Viability of ascospores was checked in an agar lid assay. In this, the lid of a petri dish was covered with a thin layer of CM agar and placed on a plate that contained inoculated wheat nodes. Forcibly discharged ascospores landed on the agar cover, where new colonies were growing. Growth assays were performed using the wild type and three independent $\Delta F g O S$-2 and Fgatfl mutant strains. Mycelial plugs taken 
from the edge of a 3-day-old colony on CM (1 liter of CM contained $10 \mathrm{ml}$ of solution $\mathrm{A}\left[\mathrm{Ca}\left(\mathrm{NO}_{3}\right)_{2} \times 4 \mathrm{H}_{2} \mathrm{O}\right.$ at $100 \mathrm{~g}$ liter $\left.{ }^{-1}\right], 10 \mathrm{ml}$ of solution $\mathrm{B}\left[\mathrm{KH}_{2} \mathrm{PO}_{4}\right.$ at $20 \mathrm{~g} \mathrm{liter}^{-1}, \mathrm{MgSO}_{4} \times$ $7 \mathrm{H}_{2} \mathrm{O}$ at $25 \mathrm{~g} \mathrm{liter}^{-1}$, and $\mathrm{NaCl}$ at $10 \mathrm{~g} \mathrm{liter}^{-1}$, sterilized by filtration], $10 \mathrm{~g}$ of glucose, $1 \mathrm{ml}$ of suspension $\mathrm{D}\left[\mathrm{H}_{3} \mathrm{BO}_{3}\right.$ at 60 g liter ${ }^{-1}, \mathrm{CuSO}_{4} \times 5 \mathrm{H}_{2} \mathrm{O}$ at $390 \mathrm{mg} \mathrm{liter}^{-1}, \mathrm{KI}$ at $13 \mathrm{mg} \mathrm{liter}^{-1}$, $\mathrm{MnSO}_{4} \times \mathrm{H}_{2} \mathrm{O}$ at $60 \mathrm{mg}$ liter ${ }^{-1},\left(\mathrm{NH}_{4}\right)_{6} \mathrm{Mo}_{7} \mathrm{O}_{24} \times 4 \mathrm{H}_{2} \mathrm{O}$ at 51 mg liter ${ }^{-1}, \mathrm{ZnSO}_{4} \times 7 \mathrm{H}_{2} \mathrm{O}$ at 5.48 g liter $^{-1}$, and $\mathrm{FeCl}_{3} \times 6 \mathrm{H}_{2} \mathrm{O}$ at $932 \mathrm{mg} \mathrm{liter}^{-1}$ ], $1 \mathrm{~g}$ of yeast extract, $0.5 \mathrm{~g}$ of enzymatically hydrolyzed casein, and $0.5 \mathrm{~g}$ of acid-hydrolyzed casein) and placed in the middle of the assay plates. The growth assay plates were supplemented with the following agents: D-sorbitol, $\mathrm{NaCl}, \mathrm{KCl}, \mathrm{H}_{2} \mathrm{O}_{2}$, and fludioxonil at concentrations as indicated in the figures and captions. The plates were inoculated at $28^{\circ} \mathrm{C}$ for at least 3 days in the dark. The diameter of the colonies was measured using a technical ruler. The analyses were performed with at least five replicates. For RNA isolation, the strains were precultured in liquid yeast-peptone-dextrose (YPD) medium for 3 days. Mycelia were harvested and washed with distilled water several times. Equal portions of semidried mycelia were then shifted in new liquid $\mathrm{CM}$ containing no additional supplement, $\mathrm{NaCl}$, or $\mathrm{H}_{2} \mathrm{O}_{2}$ at the concentrations indicated in the figure captions. Mycelia raised in the toxin induction media (discussed below) were also used for the RNA isolation.

\section{Vector construction \\ for deletion and overexpression of Fgatfl.}

Standard recombinant DNA methods were performed according to Ausubel and associates (2002) and Sambrook and associates (1989). The complete ORF of Fgatfl was deleted using a double homologous recombination approach. For vector construction, flanking regions $(1.054 \mathrm{~kb}$ upstream and $1.056 \mathrm{~kb}$ downstream of Fgatfl) were cloned by PCR from genomic DNA (gDNA) using the primers listed in Supplementary Table S5. The PCR was initiated by denaturation at $94^{\circ} \mathrm{C}$ for $4 \mathrm{~min}$, followed by 35 cycles of $94^{\circ} \mathrm{C}$ for $45 \mathrm{~s}, 55^{\circ} \mathrm{C}$ for $45 \mathrm{~s}$, and $72^{\circ} \mathrm{C}$ for $60 \mathrm{~s}$. The PCR included a final extension step at $72^{\circ} \mathrm{C}$ for $10 \mathrm{~min}$ and a cooling step at $4^{\circ} \mathrm{C}$. The replacement vector was constructed using fusion PCR. The PCR amplification was performed using the following parameters: initial denaturation at $94^{\circ} \mathrm{C}$ for $4 \mathrm{~min}$; followed by 20 cycles of $94^{\circ} \mathrm{C}$ for $45 \mathrm{~s}$, $58^{\circ} \mathrm{C}$ for $2 \mathrm{~min}$, and $72^{\circ} \mathrm{C}$ for $4 \mathrm{~min}$; and a final extension step at $72^{\circ} \mathrm{C}$ for $10 \mathrm{~min}$. The upstream and downstream fragments (200 ng each), together with the HPH cassette (hygromycin cassette; $1.8 \mathrm{~kb}$ ) released from the pGEM-HPH vector (A. Lösch, University of Hamburg, Germany) by digestion (400 ng), were used in a $25-\mu \mathrm{l}$ PCR reaction without the addition of primers because the fragments acted as primers and templates for the elongation. The PCR product was ligated into the pGEM-T vector (Promega Corp., Madison, WI, U.S.A.) to generate pGEM-Fgatf1-HPH; this was then transformed into XL1-blue Escherichia coli-competent cells (DNA Cloning Services, Biozentrum Klein Flottbek, Hamburg, Germany). Bacteria were cultivated in sterile lysogeny broth medium (Sambrook et al. 1989) either as a liquid culture or on agar plates. For the fungal transformation (discussed below), pGEM-Fgatfl-HPH was digested with $X b a \mathrm{I}$ to release the disruption construct. The vector pII99::dsRed was used for the generation of dsRedfluorescent strains (Ilgen et al. 2009). This vector facilitates the constitutive expression of dsRed under the control of the gpdA promoter of $A$. nidulans and confers geneticin resistance.

For constitutive expression of Fgatfl controlled by the gpdA from Cochliobolus heterostrophus, the ORF of Fgatfl was amplified by PCR from gDNA using the Pfu proofreading polymerase (Promega Corp., Mannheim, Germany) primers 11 and 12. The PCR product was ligated into pJET1.2 vector (Fisher Scientific, Schwerte, Germany) and sequenced. The ORF
( $1,898 \mathrm{bp})$ was excised from pJET1.2 by restriction enzymes SacI and XbaI and cloned into pII99 vector, which contains the gpdA promoter from $C$. heterostrophus, and a geneticinresistance cassette. The resulting plasmid, pII99-Fgatfl, was linearized at the unique ApaI restriction site which cuts the Fgatfl sequence at position 1,050 to facilitate single crossover events. The linearized construct was used for fungal transformation (discussed below).

For generation of FgOS-2 deletion mutants in an Fgatfl overexpression mutant, Fgatf ${ }^{\mathrm{oe}}$ mutant number 1.2 was chosen for $\mathrm{FgOS}$-2 deletion. The construct and method for $\mathrm{FgOS}$ 2 deletion is described by Nguyen and associates (2012).

\section{BiFC experiments,}

For BiFC experiments, FgOS-2 and Fgatfl were tagged with separated halves of the YFP. To fuse the N-terminal half of YFP to $F g O S$-2, a $1.074-\mathrm{kb} F g O S$-2 PCR product was amplified using primers 13 (which introduces an $N c o$ I restriction site before the starting ATG) and 14 (which introduces an NotI restriction site after the stop codon). To fuse the C-terminal part of YFP to Fgatfl, primers 15 (which introduces an NcoI restriction site before the starting ATG) and 16 (which introduces a NotI restriction site after the stop codon) were used to generate a 1.581-kb PCR product. Both products were cloned into pJET1.2 plasmids and subsequently released by restriction using enzymes $N c o I$ and NotI. The fragments were ligated into the vectors YFP-N and YFP-C (Hoff and Kück 2005) to generate plasmid YFP-N::FgOS-2 and YFP-C::Fgatf1, respectively. Both plasmids were linearized by EcoRI (YFP-N::FgOS-2) and XmnI (YFP-C::Fgatfl) and subsequently co-transformed into $F$. graminearum (discussed below).

\section{Transformation of $\boldsymbol{F}$. graminearum.}

DNA (10 to $30 \mu \mathrm{g}$ dissolved in 30 to $50 \mu$ of distilled $\mathrm{H}_{2} \mathrm{O}$ ) was used for protoplast transformation of $F$. graminearum. The protoplast transformation method was performed as previously described (Jansen et al. 2005; Proctor et al. 1995). Yeast-peptone-glucose medium $(100 \mathrm{ml} ; 0.3 \%$ yeast extract, $1 \%$ Bacto peptone, and $2 \% \mathrm{D}$-glucose) was inoculated with $1 \times 10^{6} \mathrm{co}-$ nidia and incubated overnight on a shaker (New Brunswick Innova 44, Eppendorf, Wesseling-Berzdorf, Germany) at $28^{\circ} \mathrm{C}$ and $150 \mathrm{rpm}$. The mycelia were collected by filtering with a 200- $\mu \mathrm{m}$-diameter sieve and washed with double-distilled water. Mycelia were resuspended in a $20-\mathrm{ml}$ mixture of driselase and lysing enzymes $(2.5 \%$; $0.5 \%$ in $0.6 \mathrm{M}$ or $1.2 \mathrm{M}$ $\mathrm{KCl}$ ) (Life Technologies, Darmstadt, Germany) and incubated for 2 to $3 \mathrm{~h}$ on a shaker (Eppendorf) at $30^{\circ} \mathrm{C}$ and $80 \mathrm{rpm}$. Undigested hyphal material was removed from the protoplast suspension by subsequent filtration. The protoplasts were pelleted by centrifugation at $670 \times g$, washed once with $10 \mathrm{ml}$ of $20 \%$ sucrose, $10 \mathrm{mM}$ Tris- $\mathrm{HCl}$ ( $\mathrm{pH} \mathrm{8.0)}$, and $50 \mathrm{mM} \mathrm{CaCl}_{2}$ (STC), centrifuged again, and then resuspended and adjusted in STC at $1 \times 10^{8}$ protoplasts $/ \mathrm{ml}$. For transformation, $200 \mu \mathrm{l}$ of the protoplast suspension was mixed with DNA. The samples were incubated at room temperature for $20 \mathrm{~min}$. Subsequently, $1 \mathrm{ml}$ of $40 \%$ polyethylene glycol 4000 and $60 \%$ STC was added and again incubated at room temperature for $20 \mathrm{~min}$. The protoplast suspension was added to $5 \mathrm{ml}$ of $100 \mathrm{~g}$ of sucrose, $0.3 \%$ yeast extract, and $0.3 \%$ casamino acid (TB3) medium and shaken overnight on a biological shaker (Eppendorf) at room temperature and $100 \mathrm{rpm}$ for cell wall regeneration. The regenerated protoplasts were pelleted by centrifugation at 4,200 $\times g$ and, then, mixed with TB3 agar (1.5\%) at $50^{\circ} \mathrm{C}$ with hygromycin $\mathrm{B}\left(250 \mu \mathrm{g} \mathrm{ml}^{-1}\right)$ or geneticin $(100 \mu \mathrm{g}$ $\left.\mathrm{ml}^{-1}\right)$. The mixture was then plated out on petri dishes $(10$ $\mathrm{ml} / \mathrm{plate})$. After $24 \mathrm{~h}$, an overlay comprising of TB3 agar (1.5\%) and hygromycin B at $500 \mu \mathrm{g} \mathrm{ml}^{-1}$ or geneticin $\left(100 \mu \mathrm{g} \mathrm{ml}^{-1}\right)$ was 
added to the plates. Putative transformants were obtained after $2 \mathrm{dpi}$ at $28^{\circ} \mathrm{C}$. They were transferred to fresh plates of $\mathrm{CM}$ supplemented with hygromycin B at $250 \mu \mathrm{g} \mathrm{ml}^{-1}$ or geneticin $\left(200 \mu \mathrm{g} \mathrm{ml}^{-1}\right)$ and incubated at $28^{\circ} \mathrm{C}$. The transformants were purified by single-spore isolation and subsequently checked by diagnostic PCR.

\section{Southern blot analysis.}

For Southern hybridization analysis, approximately $3 \mu \mathrm{g}$ of gDNA of the wild type and Fgatfl mutant strains was restricted with appropriate restriction enzymes overnight. The digested DNA was then separated on $0.8 \%$ agarose gel by electrophoresis at $70 \mathrm{~V}$ for 6 to $7 \mathrm{~h}$. Then, the DNA was transferred by capillary blotting onto a Hybond NX membrane (GE Healthcare, Munich) and hybridized with a digoxygenin-labeled (Roche, Penzberg, Germany) DNA internal probe, which was generated with the gene-specific primers 7 and 8. Detection and visualization procedures were carried out following the manufacturer's manual (Roche). In order to confirm single-copy integration of the hygromycin cassette, rehybridization with a hygromycin probe was performed.

\section{Virulence assays on wheat, maize, and $B$. distachyon.}

The susceptible spring wheat 'Nandu' (Lochow-Petkus, Bergen-Wohlde, Germany) and the partially resistant wheat 'Amaretto' (Berthold Bauer, Niedertraubling, Germany) were used for wheat virulence assay. Plants were cultivated in a growth room at $20^{\circ} \mathrm{C}$ with a photoperiod of $16 \mathrm{~h}$ and $60 \%$ relative humidity, then transferred to infection chambers with optimized conditions. A suspension of 500 conidia in $10 \mu \mathrm{l}$ of the wild type and all mutants was inoculated into each of two central spikelets at the early stages of anthesis (Jenczmionka and Schäfer 2005). The inoculated spikes were enclosed in small plastic bags misted with water for the first 3 days and then monitored for up to 3 weeks in the infection chambers. Wheat spikes inoculated with $10 \mu \mathrm{l}$ of pure water were used as the negative control. The wheat infection assay was performed using three independent knock-out strains, two independent overexpression mutant strains, and 10 replicates in parallel with the wild type. The maize inbred line A188 (Green and Phillips, 1975) was grown in the greenhouse (temperature, 26 to $30^{\circ} \mathrm{C}$; humidity, 70 to $85 \%$; and natural daily photoperiod with additional artificial light when required). Before inoculation, the silks were manually pollinated to ensure optimal pollination. Each maize cob was inoculated by injecting conidia suspensions into the silk channel of primary ears using a syringe and cannula (Reid et al. 1995). The infection procedure was performed with $4 \mathrm{ml}$ of conidial suspension at a concentration of the wild type and the mutant strains, each at $2 \times 10^{4}$ conidia/ml. Maize cobs inoculated with $4 \mathrm{ml}$ of pure water were used for the negative control. The inoculated cobs were enclosed in plastic bags for the first 3 days and the inoculation lasted for 5 weeks.

Brachypodium distachyon (diploid inbred line $\mathrm{Bd} 21$ ) was cultivated in a growth room at $22^{\circ} \mathrm{C}$ with a 20 -h photoperiod and then transferred to infection chambers at $22^{\circ} \mathrm{C}, 65 \%$ humidity, and a photoperiod of $8 \mathrm{~h}$. A suspension of 50 conidia $(1 \mu \mathrm{l})$ was inoculated into the central spikelet of each spike at the early stages of anthesis. The inoculated spikes were enclosed in small plastic bags for the first 2 days and then monitored for up to 2 weeks in the infection chambers.

\section{DON and ZEA production analysis.}

For in vitro DON production measurement, $5 \times 10^{3}$ conidia of the wild type and the Fgatfl mutant strains were precultured in YPD for 3 days. The mycelia were harvested by filtering with a $200-\mu \mathrm{m}$-diameter sieve, washed by rinsing at least three times with distilled water, and then dried on sterile Whatman paper (GE Healthcare). Then, $1 \mathrm{~g}$ of semidried mycelia was further incubated for 24 and $72 \mathrm{~h}$ in minimal medium supplemented with $5 \mathrm{mM}\left(\mathrm{NH}_{4}\right)_{2} \mathrm{SO}_{4}$ for DON induction. Each supernatant $(50 \mu \mathrm{l})$ was taken for DON measurement using a highly sensitive DON ELISA technique (RIDAscreen DON kits; R-Biopharm AG, Darmstadt, Germany). For the in planta analysis, four wheat spikes were each inoculated with the wild type and the mutant strains. The spikes were inoculated with pure water as the negative control. The inoculated samples were collected after $7 \mathrm{dpi}$, immediately frozen in liquid nitrogen, and dried in vacuum. Each sample $(200 \mathrm{mg})$ was ground to a powder under liquid nitrogen. Then, $50 \mathrm{mg}$ of ground material was suspended in $500 \mu \mathrm{l}$ of distilled water. The extract was manually mixed by hand or vortex and centrifuged. For the DON quantification assay, $50 \mu \mathrm{l}$ of the supernatant was used. The production of DON was measured following the manufacturer's instructions. All measurements were subsequently normalized to the amount of mycelium in the sample using quantitative polymerase chain reaction (qPCR), as previously described (Voigt et al. 2007). The sample preparation procedure for the in planta and in vitro ZEA measurements was similar to that for DON measurements, except that medium consisting of $2 \%$ yeast extract, $1.5 \%$ sucrose, $0.05 \%$ $\mathrm{MgSO}_{4} \times 7 \mathrm{H}_{2} \mathrm{O}, 1 \mathrm{ml}$ of trace metal solution, and $885 \mathrm{ml}$ of Milli Q water; $\mathrm{pH}$ 6.5) was used for in vitro ZEA induction. Quantification was performed according to the manual contained in the RIDAscreen ZEA kit (R-Biopharm AG).

\section{Catalase activity measurement and ROS detection assay.}

For the catalase activity measurement, the wild type, $\Delta F g O S-2$, and $\Delta F g a t f 1$ were precultured in YPD and then shifted to liquid $\mathrm{CM}$ with and without addition of $0.8 \mathrm{M} \mathrm{NaCl}$ and $10 \mathrm{mM} \mathrm{H}_{2} \mathrm{O}_{2}$. After $2 \mathrm{~h}$ of exposure, mycelia were harvested and then mixed with extraction buffer $(150 \mathrm{mM} \mathrm{KCl}$, $200 \mathrm{mM}$ EDTA, and $50 \mathrm{mM} \mathrm{K}_{2} \mathrm{PO}_{4}, \mathrm{pH}$ 7.4). The resulting homogenate was centrifuged at $16,100 \times g$ for $20 \mathrm{~min}$ at $4^{\circ} \mathrm{C}$, and the supernatant was used for enzyme assay. Total catalase activity was measured using the Amplex red catalase assay kit (Life Technologies) according to the manufacturer's instructions. Catalase activity was normalized to the amount of total protein content determined by Bradford reagent (Sigma, Taufkirchen, Germany). The superoxide production in whole fungal colonies was detected by staining with NBT (Sigma). The wild type and $\Delta F g O S-2$ and Fgatfl mutant strains were cultured on $\mathrm{CM}$ and $\mathrm{CM}$ agar plates, which were supplemented with $0.2 \mathrm{M} \mathrm{NaCl}$ or $0.8 \mathrm{M} \mathrm{NaCl}$ at $28^{\circ} \mathrm{C}$ in the dark for 3 days. Then, each plate was stained with $20 \mathrm{ml}$ of $0.2 \%$ (wt/vol) NBT solution (NBT diluted in water) and incubated in the dark at $28^{\circ} \mathrm{C}$ for 30 to 45 min. The supernatant was drained and the plates were washed with $99 \%$ ethanol. The plates were reincubated for 30 to 45 min in the dark at $28^{\circ} \mathrm{C}$ before images of whole colonies were taken.

\section{Expression analysis by semiquantitative reverse-transcriptase PCR and real-time qPCR.}

In order to study the expression of possible targets of FgOS-2 and FgAtf1 in $F$. graminearum in vitro and in planta, RNA was isolated using a Nucleospin RNA II Kit (Macherey-Nagel, Düren, Germany). Samples were collected from in vitro submersed cultures and from wheat spikes at 3 and $7 \mathrm{dpi}$, respectively, and frozen in liquid nitrogen for subsequent RNA isolation. For reverse transcription (RT)-PCR, SuperScript II RNase $\mathrm{H}$ reverse transcriptase (Life Technologies) was used, according to the manufacturer's instructions. The resulting single-stranded cDNA was later used as a template for real-time qRT-PCR reactions. Transcript levels of the target genes were normalized 
against $\beta$-tubulin gene expression. The qRT-PCR reactions were carried out using gene-specific primers, with SYBR Green qPCR SuperMix-UDG (Thermo Scientific, Dreieich, Germany) in a volume of $20 \mu \mathrm{l}$ in a LightCycler 480 (Roche). The PCR program was as follows: incubation for $2 \mathrm{~min}$ at $50^{\circ} \mathrm{C}$, then 2 min at $95^{\circ} \mathrm{C}$, followed by up to 40 cycles of denaturation at $94^{\circ} \mathrm{C}$ for $30 \mathrm{~s}$, annealing at 55 to $58^{\circ} \mathrm{C}$ for $30 \mathrm{~s}$, and extension at $72^{\circ} \mathrm{C}$ for $15 \mathrm{~s}$, followed by a melting curve analysis in order to check the specificity of fragment amplification. All of the measurements were repeated twice, each with three replicates. Relative changes in gene expression were calculated using the comparative cycle threshold method (using LC 480 software for the LightCycler 480). The PCR reactions were conducted with specific primers. The level of constitutively expressed $\beta$-tubulin mRNA served as reference.

\section{Microscopic analysis of the germinating conidia, BiFC assays, and FgAtf1 localization studies.}

For microscopic analysis of the germinating conidia, the wild type and $\Delta F$ gatfl strains were precultured in a liquid $\mathrm{CM}$ and a CM supplemented with $0.8 \mathrm{M} \mathrm{NaCl}$ for $16 \mathrm{~h}$ and then centrifuged for $15 \mathrm{~min}$ at $16,100 \times g$. The pellets were rinsed and diluted again with double-distilled water. Samples were placed on glass slides and analyzed under a Zeiss microscope Axio Imager Z1 (Carl Zeiss, Jena, Germany). For BiFC assays, conidia of the wild type and BiFC strains were precultured in liquid $\mathrm{CM}$ for $16 \mathrm{~h}$ and then centrifuged and washed with double-distilled water to remove liquid CM. Mycelia were treated with $1.2 \mathrm{M} \mathrm{NaCl}$ and examined by confocal laserscanning microscopy with filter sets for excitation at $514 \mathrm{~nm}$ (YFP) and $594 \mathrm{~nm}$ (dsRed) and emission wavelength detection from 519 to $573 \mathrm{~nm}$ (YFP) and 588 to $740 \mathrm{~nm}$ (dsRed). Untreated samples served as the negative control. For microscopy of the infection progress in wheat, inoculated wheat heads were manually harvested after 7 and 21 dpi and cut longitudinally using a razor blade. Red fluorescence of fungal mycelium was visualized using a Leica MZ FLIII (Leica Microsystems, Heerbrugg, Switzerland) equipped with a 545- to 530-nm excitation and a 620- to 660-nm BP emission filter set. To stain the nuclei, strains were cultured in a liquid $\mathrm{CM}$ for $16 \mathrm{~h}$, then centrifuged for $15 \mathrm{~min}$ at $16,100 \times g$. The pellets were rinsed and diluted again with distilled water prior to staining. Samples were placed on glass slides and stained with 4',6-diamidino-2phenylindole at $1 \mu \mathrm{g} \mathrm{ml}^{-1}$ for $10 \mathrm{~min}$ before being analyzed under a Zeiss microscope Axio Imager Z1 equipped with filter sets for excitation at 538 to $562 \mathrm{~nm}$ and emission wavelength detection from 570 to $640 \mathrm{~nm}$.

\section{ACKNOWLEDGMENTS}

We thank B. Hadeler for technical assistance, M. J. Boenisch for assistance with the microscopy, B. Doormann for critically reading the manuscript, and Hong Duc University, Vietnam, for their support and cooperation.

\section{LITERATURE CITED}

Albertyn, J., Hohmann, S., Thevelein, J. M., and Prior, B. A. 1994. GPD1, which encodes glycerol-3-phosphate dehydrogenase, is essential for growth under osmotic stress in Saccharomyces cerevisiae, and its expression is regulated by the high-osmolarity glycerol response pathway. Mol. Cell. Biol. 14:4135-4144.

Apel, K., and Hirt, H. 2004. Reactive oxygen species: Metabolism, oxidative stress, and signal transduction. Annu. Rev. Plant Biol. 55:373-399.

Ausubel, F. M., Brent, R., Kingston, R. E., Moore, D. D., Seidman, J. G., Smith, J. A., and Struhl, K. 2002. Short Protocols in Molecular Biology: A Compendium of Methods from Current Protocols in Molecular Biology. Wiley, New York.

Buck, V., Quinn, J., Soto Pino, T., Martin, H., Saldanha, J., Makino, K., Morgan, B. A., and Millar, J. B. 2001. Peroxide sensors for the fission yeast stress-activated mitogen-activated protein kinase pathway. Mol. Biol. Cell 12:407-419.

Calvo, A. M., Wilson, R. A., Bok, J. W., and Keller, N. P. 2002. Relationship between secondary metabolism and fungal development. Microbiol. Mol. Biol. Rev. 66:447-459.

Cano-Domínguez, N., Alvarez-Delfin, K., Hansberg, W., and Aguirre, J. 2008. NADPH oxidases NOX-1 and NOX-2 require the regulatory subunit NOR-1 to control cell differentiation and growth in Neurospora crassa. Eukaryot. Cell 7:1352-1361.

Chen, D., Toone, W. M., Mata, J., Lyne, R., Burns, G., Kivinen, K., Brazma, A., Jones, N., and Bähler, J. 2003. Global transcriptional responses of fission yeast to environmental stress. Mol. Biol. Cell 14:214-229.

Colot, H. V., Park, G., Turner, G. E., Ringelberg, C., Crew, C. M., Litvinkova, L., Weiss, R. L., Borkovich, K. A., and Dunlap, J. C. 2006. A high-throughput gene knockout procedure for Neurospora reveals functions for multiple transcription factors. Proc. Natl. Acad. Sci. U.S.A. 103:10352-10357.

Davidson, M. K., Shandilya, H. K., Hirota, K., Ohta, K., and Wahls, W. P. 2004. Atf1-Pcr1-M26 complex links stress-activated MAPK and cAMP-dependent protein kinase pathways via chromatin remodeling of cgs2+. J. Biol. Chem. 279:50857-50863.

El Oirdi, M., El Rahman, T. A., Rigano, L., El Hadrami, A., Rodriguez, M. C., Daayf, F., Vojnov, A., and Bouarab, K. 2011. Botrytis cinerea manipulates the antagonistic effects between immune pathways to promote disease development in tomato. Plant Cell Online 23:2405-2421.

Finkel, T. 2003. Oxidant signals and oxidative stress. Curr. Opin. Cell Biol. 15:247-254.

Foreman, J., Demidchik, V., Bothwell, J. H., Mylona, P., Miedema, H. Torres, M. A., Linstead, P., Costa, S., Brownlee, C., Jones, J. D., Davies, J. M., and Dolan, L. 2003. Reactive oxygen species produced by NADPH oxidase regulate plant cell growth. Nature 422:442-446.

Gadjev, I., Stone, J. M., and Gechev, T. S. 2008. Programmed cell death in plants: New insights into redox regulation and the role of hydrogen peroxide. Int. Rev. Cell. Mol. Biol. 270:87-144.

Gaffoor, I., Brown, D. W., Plattner, R., Proctor, R. H., Qi, W., and Trail, F 2005. Functional analysis of the polyketide synthase genes in the filamentous fungus Gibberella zeae (anamorph Fusarium graminearum). Eukaryot. Cell 4:1926-1933.

Gardiner, D. M., Kazan, K., and Manners, J. M. 2009a. Nutrient profiling reveals potent inducers of trichothecene biosynthesis in Fusarium graminearum. Fungal Genet. Biol. 46:604-613.

Gardiner, D. M., Kazan, K., and Manners, J. M. 2009b. Novel genes of Fusarium graminearum that negatively regulate deoxynivalenol production and virulence. Mol. Plant-Microbe Interact. 22:1588-1600.

Gardiner, D. M., Osborne, S., Kazan, K., and Manners, J. M. 2009c. Low $\mathrm{pH}$ regulates the production of deoxynivalenol by Fusarium graminearum. Microbiology 155:3149-3156.

Gechev, T. S., Van Breusegem, F., Stone, J. M., Denev, I., and Laloi, C. 2006. Reactive oxygen species as signals that modulate plant stress responses and programmed cell death. BioEssays 28:1091-1101.

Giesbert, S., Schürg, T., Scheele, S., and Tudzynski, P. 2008. The NADPH oxidase Cpnox 1 is required for full pathogenicity of the ergot fungus Claviceps purpurea. Mol. Plant Pathol. 9:317-327.

Govrin, E. M., and Levine, A. 2000. The hypersensitive response facilitates plant infection by the necrotrophic pathogen Botrytis cinerea. Curr. Biol. 10:751-757.

Green, C. E., and Phillips, R. L. 1975. Plant regeneration from tissue cultures of maize. Crop Sci. 15:417-421.

Guo, M., Guo, W., Chen, Y., Dong, S., Zhang, X., Zhang, H., Song, W., Wang, W., Wang, Q., Lv, R., Zhang, Z., Wang, Y., and Zheng, X. 2010. The basic leucine zipper transcription factor Moatf1 mediates oxidative stress responses and is necessary for full virulence of the rice blast fungus Magnaporthe oryzae. Mol. Plant-Microbe Interact. 23:10531068 .

Harris, L. J., Desjardins, A. E., Plattner, R. D., Nicholson, P., Butler, G., Young, J. C., Weston, G., Proctor, R. H., and Hohn, T. M. 1999. Possible role of trichothecene mycotoxins in virulence of Fusarium graminearum on maize. Plant Dis. 83:954-960.

Heller, J., and Tudzynski, P. 2011. Reactive Oxygen Species in phytopathogenic fungi: Signaling, development, and disease. Annu. Rev. Phytopathol. 49:369-390

Heller, J., Ruhnke, N., Espino, J. J., Massaroli, M., Collado, I. G., and Tudzynski, P. 2012. The mitogen-activated protein kinase BcSak1 of Botrytis cinerea is required for pathogenic development and has broad regulatory functions beyond stress response. Mol. Plant-Microbe Interact. 25:802-816.

Hoff, B., and Kück, U. 2005. Use of bimolecular fluorescence complementation to demonstrate transcription factor interaction in nuclei of living cells from the filamentous fungus Acremonium chrysogenum. Curr. Genet. 47:132-138. 
Hohmann, S. 2002. Osmotic stress signaling and osmoadaptation in yeasts. Microbiol. Mol. Biol. Rev. 66:300-372.

Ilgen, P., Hadeler, B., Maier, F. J., and Schäfer, W. 2009. Developing kernel and rachis node induce the trichothecene pathway of Fusarium graminearum during wheat head infection. Mol. Plant-Microbe Interact. 22:899-908.

Jansen, C., von Wettstein, D., Schäfer, W., Kogel, K. H., Felk, A., and Maier, F. J. 2005. Infection patterns in barley and wheat spikes inoculated with wild-type and trichodiene synthase gene disrupted Fusarium graminearum. Proc. Natl. Acad. Sci. U.S.A. 102:16892-16897.

Jenczmionka, N. J., and Schäfer, W. 2005. The Gpmk1 MAP kinase of Fusarium graminearum regulates the induction of specific secreted enzymes. Curr. Genet. 47:29-36.

Jenczmionka, N. J., Maier, F. J., Lösch, A. P., and Schäfer, W. 2003. Mating, conidiation and pathogenicity of Fusarium graminearum, the main causal agent of the head-blight disease of wheat, are regulated by the MAP kinase Gpmk1. Curr. Genet. 43:87-95.

Ježek, P., and Hlavatá, L. 2005. Mitochondria in homeostasis of reactive oxygen species in cell, tissues, and organism. Int. J. Biochem. Cell B 37:2478-2503.

Jiang, J., Yun, Y., Fu, J., Shim, W.-B., and Ma, Z. 2011. Involvement of a putative response regulator FgRrg-1 in osmotic stress response, fungicide resistance and virulence in Fusarium graminearum. Mol. Plant Pathol. 12:425-436.

Jiao, F., Kawakami, A., and Nakajima, T. 2008. Effects of different carbon sources on trichothecene production and Tri gene expression by Fusarium graminearum in liquid culture. FEMS (Fed. Eur. Microbiol. Soc.) Microbiol. Lett. 285:212-219.

Kawasaki, L., Sanchez, O., Shiozaki, K., and Aguirre, J. 2002. SakA MAP kinase is involved in stress signal transduction, sexual development and spore viability in Aspergillus nidulans. Mol. Microbiol. 45:1153-1163.

Keller, N. P., and Hohn, T. M. 1997. Metabolic pathway gene clusters in filamentous fungi. Fungal Genet. Biol. 21:17-29.

Kim, J. E., Han, K. H., Jin, J., Kim, H., Kim, J. C., Yun, S. H., and Lee, Y W. 2005. Putative polyketide synthase and laccase genes for biosynthesis of aurofusarin in Gibberella zeae. Appl. Environ. Microbiol. 71:17011708 .

Kuiper-Goodman, T., Scott, P. M., and Watanabe, H. 1987. Risk assessment of the mycotoxin zearalenone. Regul. Toxicol. Pharmacol. 7:253306.

Lamb, T. M., Finch, K. E., and Bell-Pedersen, D. 2012. The Neurospora crassa OS MAPK pathway-activated transcription factor ASL-1 contributes to circadian rhythms in pathway responsive clock-controlled genes. Fungal Genet. Biol. 49:180-188.

Lara-Ortiz, T., Riveros-Rosas, H., and Aguirre, J. 2003. Reactive oxygen species generated by microbial NADPH oxidase NoxA regulate sexual development in Aspergillus nidulans. Mol. Microbiol. 50:12411255 .

Lara-Rojas, F., Sánchez, O., Kawasaki, L., and Aguirre, J. 2011. Aspergillus nidulans transcription factor AtfA interacts with the MAPK SakA to regulate general stress responses, development and spore functions. Mol. Microbiol. 80:436-454.

Linnemannstöns, P., Schulte, J., del Mar Prado, M., Proctor, R. H., Avalos, J., and Tudzynski, B. 2002. The polyketide synthase gene pks4 from Gibberella fujikuroi encodes a key enzyme in the biosynthesis of the red pigment bikaverin. Fungal Genet. Biol. 37:134-148.

Maier, F. J., Miedaner, T., Hadeler, B., Felk, A., Salomon, S., Lemmens, M., Kassner, H., and Schäfer, W. 2006. Involvement of trichothecenes in fusarioses of wheat, barley and maize evaluated by gene disruption of the trichodiene synthase (Tri5) gene in three field isolates of different chemotype and virulence. Mol. Plant Pathol. 7:449-461.

Malagnac, F., Lalucque, H., Lepere, G., and Silar, P. 2004. Two NADPH oxidase isoforms are required for sexual reproduction and ascospore germination in the filamentous fungus Podospora anserina. Fungal Genet. Biol. 41:982-997.

Malz, S., Grell, M. N., Thrane, C., Maier, F. J., Rosager, P., Felk, A., Albertsen, K. S., Salomon, S., Bohn, L., Schäfer, W., and Giese, H. 2005. Identification of a gene cluster responsible for the biosynthesis of aurofusarin in the Fusarium graminearum species complex. Fungal Genet. Biol. 42:420-433.

Medentsev, A. G., Kotik, A. N., Trufanova, V. A., and Akimenko, V. K. 1993. Identification of aurofusarin in Fusarium graminearum isolates, causing a syndrome of worsening of egg quality in chickens. Prikl. Biokhim. Mikrobiol. 29:542-546.

Molina, L., and Kahmann, R. 2007. An Ustilago maydis gene involved in $\mathrm{H}_{2} \mathrm{O}_{2}$ detoxification is required for virulence. Plant Cell Online 19:2293-2309.

Moye-Rowley, W. S. 2003. Regulation of the transcriptional response to oxidative stress in fungi: Similarities and differences. Eukaryot. Cell 2:381-389.
Nathues, E., Joshi, S., Tenberge, K. B., von den Driesch, M., Oeser, B Baumer, N., Mihlan, M., and Tudzynski, P. 2004. CPTF1, a CREB-like transcription factor, is involved in the oxidative stress response in the phytopathogen Claviceps purpurea and modulates ROS level in its host Secale cereale. Mol. Plant-Microbe Interact. 17:383-393.

Navarro, R. E., Stringer, M. A., Hansberg, W., Timberlake, W. E., and Aguirre, J. 1996. catA, a new Aspergillus nidulans gene encoding a developmentally regulated catalase. Curr. Genet. 29:352-359.

Nguyen, A. N., Lee, A., Place, W., and Shiozaki, K. 2000. Multistep phosphorelay proteins transmit oxidative stress signals to the fission yeast stress-activated protein kinase. Mol. Biol. Cell 11:1169-1181.

Nguyen, T. V., Schäfer, W., and Bormann, J. 2012. The stress-activated protein kinase FgOS-2 is a key regulator in the life cycle of the cereal pathogen Fusarium graminearum. Mol. Plant-Microbe Interact. 25:1142-1156.

Ochiai, N., Tokai, T., Nishiuchi, T., Takahashi-Ando, N., Fujimura, M. and Kimura, M. 2007. Involvement of the osmosensor histidine kinase and osmotic stress-activated protein kinases in the regulation of secondary metabolism in Fusarium graminearum. Biochem. Biophys. Res. Commun. 363:639-644.

Orozco-Cárdenas, M. L., Narváez-Vásquez, J., and Ryan, C. A. 2001. Hydrogen peroxide acts as a second messenger for the induction of defense genes in tomato plants in response to wounding, systemin, and methyl jasmonate. Plant Cell Online 13:179-191.

Paris, S., Wysong, D., Debeaupuis, J. P., Shibuya, K., Philippe, B., Diamond, R. D., and Latge, J. P. 2003. Catalases of Aspergillus fumigatus. Infect. Immun. 71:3551-3562.

Pascual-Ahuir, A., Serrano, R., and Proft, M. 2001. The Sko1p repressor and Gcn4p activator antagonistically modulate stress-regulated transcription in Saccharomyces cerevisiae. Mol. Cell. Biol. 21:16-25.

Passardi, F., Penel, C., and Dunand, C. 2004. Performing the paradoxical: How plant peroxidases modify the cell wall. Trends Plant Sci. 9:534 540.

Pillonel, C., and Meyer, T. 1997. Effect of phenylpyrroles on glycerol accumulation and protein kinase activity of Neurospora crassa. Pestic. Sci. 49:229-236.

Ponts, N., Pinson Gadais, L., Verdal Bonnin, M. N., Barreau, C., and Richard-Forget, F. 2006. Accumulation of deoxynivalenol and its 15 acetylated form is significantly modulated by oxidative stress in liquid cultures of Fusarium graminearum. FEMS (Fed. Eur. Microbiol. Soc.) Microbiol. Lett. 258:102-107.

Proctor, R. H., Hohn, T. M., and McCormick, S. P. 1995. Reduced virulence of Gibberella zeae caused by disruption of a trichothecene toxin biosynthetic gene. Mol. Plant-Microbe Interact. 8:593-601.

Reid, L. M., Hamilton, R. I., and Mather, D. E. 1995. Effect of macroconidial suspension volume and concentration on expression of resistance to Fusarium graminearum in maize. Plant Dis. 79:461-466.

Reiter, W., Watt, S., Dawson, K., Lawrence, C. L., Bahler, J., Jones, N., and Wilkinson, C. R. 2008. Fission yeast MAP kinase Sty1 is recruited to stress-induced genes. J. Biol. Chem. 283:9945-9956.

Rocha, O., Ansari, K., and Doohan, F. M. 2005. Effects of trichothecene mycotoxins on eukaryotic cells: A review. Food Addit. Contam. 22:369378.

Salomon, S., Gácser, A., Frerichmann, S., Kröger, C., Schäfer, W., and Voigt, C. A. 2012. The secreted lipase FGL1 is sufficient to restore the initial infection step to the apathogenic Fusarium graminearum MAP kinase disruption mutant $\Delta$ gpmk1. Eur. J. Plant Pathol. 134:23-37.

Sambrook, J., Russell, D. W., Janssen, K., and Argentine, J. 1989. Molecular Cloning: A Laboratory Manual. Cold Spring Harbor Laboratory Press, Cold Spring Harbor, NY, U.S.A.

Scott, B., and Eaton, C. J. 2008. Role of reactive oxygen species in fungal cellular differentiations. Curr. Opin. Microbiol. 11:488-493.

Segmüller, N., Ellendorf, U., Tudzynski, B., and Tudzynski, P. 2007. BcSAK1, a stress-activated mitogen-activated protein kinase, is involved in vegetative differentiation and pathogenicity in Botrytis cinerea. Eukaryot. Cell 6:211-221.

Sigrist, C. J., Cerutti, L., de Castro, E., Langendijk-Genevaux, P. S., Bulliard, V., Bairoch, A., and Hulo, N. 2010. PROSITE, a protein domain database for functional characterization and annotation. Nucleic Acids Res. 38:D161-166

Takada, H., Nishimura, M., Asayama, Y., Mannse, Y., Ishiwata, S., Kita, A., Doi, A., Nishida, A., Kai, N., Moriuchi, S., Tohda, H., Giga-Hama, Y., Kuno, T., and Sugiura, R. 2007. Atf1 is a target of the mitogen-activated protein kinase Pmk1 and regulates cell integrity in fission yeast. Mol. Biol. Cell 18:4794-4802.

Tanaka, A., Christensen, M. J., Takemoto, D., Park, P., and Scott, B. 2006 Reactive oxygen species play a role in regulating a fungus-perennial ryegrass mutualistic interaction. Plant Cell 18:1052-1066.

Temme, N., Oeser, B., Massaroli, M., Heller, J., Simon, A., Gonzalez Collado, I., Viaud, M., and Tudzynski, P. 2012. BcAtf1, a global regula- 
tor, controls various differentiation processes and phytotoxin production in Botrytis cinerea. Mol. Plant Pathol. 13:704-718.

Thannickal, V. J., and Fanburg, B. L. 2000. Reactive oxygen species in cell signaling. Am. J. Physiol. Lung C 279:L1005-L1028.

Tsuyuki, R., Yoshinari, T., Sakamoto, N., Nagasawa, H., and Sakuda, S. 2011. Enhancement of trichothecene production in Fusarium graminearum by cobalt chloride. J. Agric. Food Chem. 59:39-69.

Urban, M., Mott, E., Farley, T., and Hammond-Kosack, K. 2003. The Fusarium graminearum MAP1 gene is essential for pathogenicity and development of perithecia. Mol. Plant Pathol. 4:347-359.

Voegele, R. T., Hahn, M., Lohaus, G., Link, T., Heiser, I., and Mendgen, K. 2005. Possible roles for mannitol and mannitol dehydrogenase in the biotrophic plant pathogen Uromyces fabae. Plant Physiol. 137:190198

Voigt, C., von Scheidt, B., Gácser, A., Kassner, H., Lieberei, R., Schäfer,
W., and Salomon, S. 2007. Enhanced mycotoxin production of a lipasedeficient Fusarium graminearum mutant correlates to toxin-related gene expression. Eur. J. Plant Pathol. 117:1-12.

Wong, P., Walter, M., Lee, W., Mannhaupt, G., Münsterkötter, M., Mewes, H. W., Adam, G., and Güldener, U. 2010. FGDB: Revisiting the genome annotation of the plant pathogen Fusarium graminearum. Nucleic Acids Res. 39:637-639.

Yamashita, K., Shiozawa, A., Watanabe, S., Fukumori, F., Kimura, M., and Fujimura, M. 2008. ATF-1 transcription factor regulates the expression of ccg-1 and cat-1 genes in response to fludioxonil under OS-2 MAP kinase in Neurospora crassa. Fungal Genet. Biol. 45:1562-1569.

Zheng, D., Zhang, S., Zhou, X., Wang, C., Xiang, P., Zheng, Q., and Xu, J. R. 2012. The FgHOG1 pathway regulates hyphal growth, stress responses, and plant infection in Fusarium graminearum. PLoS One 7:e49495. Published online. 